\title{
Experimental and numerical investigation of coupled microvibration dynamics for satellite reaction wheels
}

\author{
D. Addari ${ }^{a, *}$, G. S. Aglietti ${ }^{a}$, M. Remedia ${ }^{a}$ \\ ${ }^{a}$ Surrey Space Centre, University of Surrey, Guildford, UK, GU2 7XH
}

\begin{abstract}
Microvibrations of a satellite reaction wheel assembly are commonly analysed in either hard-mounted or coupled boundary conditions, though coupled wheel-to-structure disturbance models are more representative of the real environment in which the wheel operates. This article investigates the coupled microvibration dynamics of a cantilever configured reaction wheel assembly mounted on either a stiff or flexible platform. Here a method is presented to cope with modern project necessities: (i) need of a model which gives accurate estimates covering a wide frequency range; (ii) reduce the personnel and time costs derived from the test campaign, (iii) reduce the computational effort without affecting the quality of the results. The method involves measurements of the disturbances induced by the reaction wheel assembly in a hard-mounted configuration and of the frequency and speed dependent dynamic mass of the reaction wheel. In addition, it corrects the approximation due to missing speed dependent dynamic mass in conventional reaction wheel assembly microvibration analysis. The former was evaluated experimentally
\end{abstract}

\footnotetext{
*Corresponding author, contact no. +44 7919311771

Email addresses: d.addari@surrey.ac.uk (D. Addari), g.aglietti@surrey.ac.uk
} (G. S. Aglietti), m.remedia@surrey.ac.uk (M. Remedia) 
using a previously designed and validated platform. The latter, on the other hand, was estimated analytically using a finite element model of the wheel assembly. Finally, the validation of the coupled wheel-structure disturbance model is presented, giving indication of the level of accuracy that can be achieved with this type of analyses.

Keywords: Microvibration, Dynamic Coupling, Dynamic Mass, Reaction Wheel Assembly

\section{Introduction}

Issues related to microvibration disturbances on a satellite are a major concern for missions where high levels of pointing accuracy and stringent platform stability are required [1-7]. Furthermore, it has also become of relevance for the low cost end of the market. For instance, mini- and microsatellites such as the SSTL-300-S1 platform [8, 9] or Skybox [10] have the mission to carry cameras with ground resolutions toward 1 metre hence, even small displacements (i.e. in the order of micro-metres) of the mounting interfaces of the instrument may lead to unacceptable large oscillations of the instrument line of sight. Microvibrations are classified as low level mechanical accelerations typically in the range of microgravity $(\mu g)$ usually occurring at frequencies from a few $\mathrm{Hz}$ up to several hundred $\mathrm{Hz}$ [11]. Microvibrations are generally produced by internal mechanisms on-board spacecraft, including Reaction Wheel Assemblies (RWAs), momentum wheel assemblies, cryocoolers, solar and antenna pointing mechanisms, and thrusters. Among the various disturbance sources on a spacecraft, RWAs are commonly considered as the largest $[12,13]$. The induced disturbances are transferred through the 
satellite structure towards the payloads and sensitive instruments, exciting their modes of vibration and severely affecting their performance. Moreover, estimates of the microvibration effects become more complicated as the dynamics of the microvibration sources also couple with those of the satellite structure $[14,15]$. In addition, the understanding and control of the vibration level at sensitive locations, using passive damping and active control technologies $[16,17]$, is also a crucial factor in order to achieve the desired instruments' performance.

The rotor dynamics of a Wheel Assembly (WAs) for space application in both the symmetrical and cantilevered configurations have been extensively developed in literature [18-21]. Although the two arrangements are different, they show similar dynamic behaviour, except for the two flexural modes (radial translation and in-plane rotations) which are well defined and separated for the symmetrical design, but coupled for the cantilevered configuration. In this paper, a cantilever configured RWA, illustrated in Fig. 1, is considered and a mathematical model to describe its dynamics presented. The model is then implemented with a supporting structure (either stiff or flexible) to perform microvibration coupled analyses and tests, also described herein. The first challenge towards satellite microvibration analysis is the characterisation of the potential disturbance sources. Two different methods are usually adopted for measuring the RWA-induced disturbances depending on their boundary conditions: hard-mounted configuration (isolated, with the RWA rigidly grounded on a multi-axis dynamometric platform, e.g. Kistler table) and coupled (i.e. RWA mounted on a supporting flexible structure hung free-free by elastic cords). The former has been thoroughly developed 
(a)

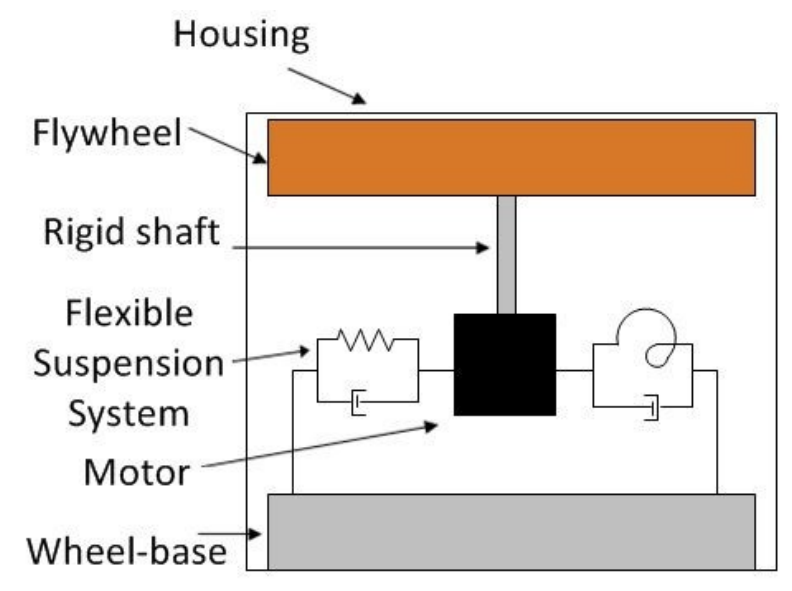

(b)

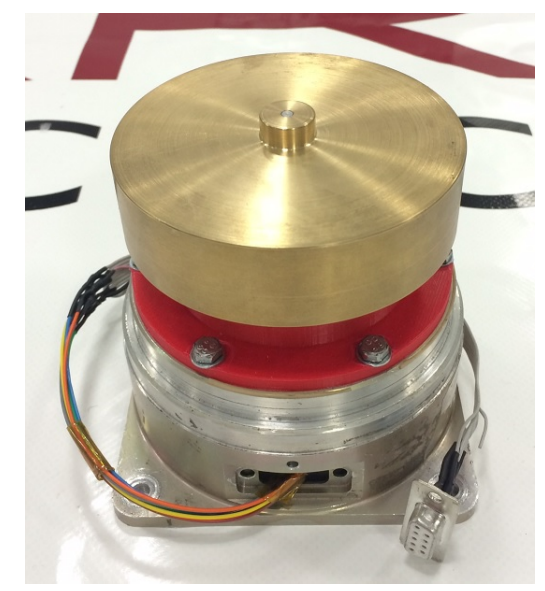

Fig. 1.

Wheel assembly model: $(a)$ cantilever configured RWA and $(b)$ cantilevered RWA used in test.

in the past, and the most representative works are reported in [19, 21-24]. However, dynamometric platforms cannot generally be used for coupled measurements, due to their size and weight. An air-floating vibration detection system was described in [24] and subsequently adopted in [25]. Although accurate, the system can operate only up to a maximum frequency of $20 \mathrm{~Hz}$. The direct measurement approach for evaluating RWA-structure coupled microvibrations is, in contrast, not as mature.

For this reason, the current practice in the space industry is to use the outcomes from the hard-mounted configuration measurements as direct inputs for the satellite microvibration analysis. Its fundamental concept is, however, flawed as hard-mounted microvibrations do not represent the real environment in which the RWAs will operate. In fact, the RWA is mounted on a "flexible" satellite structure and therefore, the loads exchanged at the in- 
terface are different from those derived from the hard-mounted configuration. With the aim to analytically reproduce the dynamics between a source and its supporting structure, the dynamic mass (or its inverse, the accelerance) of the source or the driving point accelerance of the supporting structure need to be evaluated [23].

Generally, the forces and moments obtained from hard-mounted configuration measurements are applied at the location where the source is mounted on the structure, adding a lumped inertia to include the source [23, 26-28]. This method is typically able to provide good predictions of the satellite performance if the RWA has resonance frequencies well above the frequency range of interest, which is not often the case for microvibration applications, hence the internal dynamics of the source (dynamic mass) need to be taken into account. Works to investigate and derive the dynamic mass of the source were initially carried out in $[23,29,30]$. However, all of them assumed the flywheel in a static condition (flywheel at zero speed) thus not including the gyroscopic effect. In [31], a detailed method to obtain the dynamic mass of a RWA including the gyroscopic effects was developed and validated. Although the results are accurate, this method is significantly challenging in terms of test configuration and computational effort.

In this article, the dynamic mass of the RWA, retrieved experimentally in a static condition and expanded analytically to include the gyroscopic effect by means of the RWA Finite Element (FE) model, is implemented with hard-mounted loads' measurements to estimate the coupled dynamics between a RWA and a supporting structure. The article aims to demonstrate the importance of the dynamic mass and, in particular, the improvements 
in the results due to considering both flywheel angular speed and frequency dependance.

In this study, a stiff Aluminium platform and a honeycomb structural panel are used as supporting structure for a cantilever configured RWA. The two assemblies were tested for coupled microvibration analysis hanging the system free-free using elastic cords and their characteristics, from both experimental and analytical results, are compared and discussed herein. Moreover, the agreement between the data is quantified by means of Frequency Response Assurance Criterion (FRAC)[32]. The FRAC fundamental assumption lies in the premise that the measured and synthesized data should be linearly related (unity scaling coefficient) over the full range of frequency. Finally, discussions on the level of accuracy that can be achieved with this type of analyses are provided.

\section{Mathematical Model}

In this study, a cantilever configured RWA disturbance model developed in [33] and subsequently re-elaborated in [34] using an energy approach to derive the generalised Equations of Motion (EoMs) through Lagrange's equation [35] is considered. The process is here briefly summarized for convenience of the reader. The model is subsequently implemented to predict the dynamic mass of the RWA in both static and operative conditions.

\subsection{Wheel Assembly Modelling}

The development of the complete mathematical model includes two cases: balanced flywheel and imbalanced flywheel. The simplified imbalanced wheel model is shown in Fig. 2. In order to clarify the terminology adopted in this 


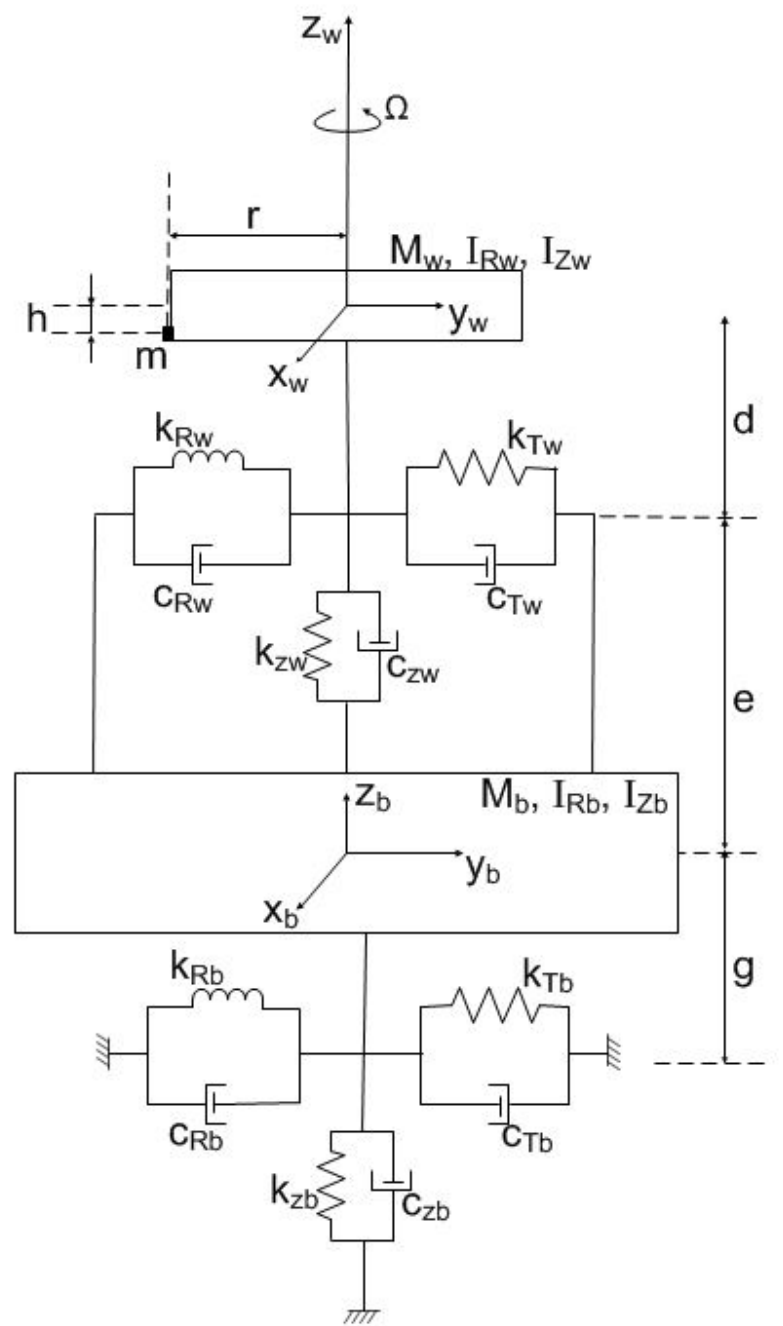

Fig. 2.

Simplified imbalance cantilever configured wheel assembly model 
paper, the parameters referring to the RWA flywheel are marked with the subscript $w$ whereas, for those relative to the RWA wheel-base, the subscript $b$ is used. The flywheel was modelled as a rigid disk with mass $M_{w}$, torsional inertia $I_{R_{w}}$, and moment of inertia w.r.t. spin axis $I_{Z_{w}}$. In addition, it was connected by a mass-less and rigid shaft of length $d$ to the soft-suspension system. The inertial frame $X_{w} Y_{w} Z_{w}$ and the body frame $x_{w} y_{w} z_{w}$ coincided at the Centre of Mass $(\mathrm{CoM})$ of the flywheel $O_{w}$ with the $z_{w^{-}}$axis (or $Z_{w^{-}}$ axis) defined in the shaft pointing direction. Rotations about the three axes in their corresponding frames are $\theta_{w}, \varphi_{w}$ and $\psi_{w}$. Let $\Omega$ be the constant flywheel rotation speed and assume the flywheel is at steady speed rotation, $\Omega=\dot{\psi}_{w}$. Similarly, the wheel-base is modelled as a rigid disk of mass $M_{b}$, radial moment of inertia $I_{R_{b}}$ and moment of inertia w.r.t. spin axis $I_{Z_{b}}$. Its $\mathrm{CoM}$ is set at a distance $e$ from the suspension system connecting the flywheel and the wheel-base and $g$ from the suspension system linking the wheel-base and the ground.

The flexible components in this system were the suspension system that connected the flywheel and the wheel-base (denoted with subscript $w$ ) and the suspension system that connected the wheel-base to the ground (denoted with subscript $b$ ). The latter was able to represent a hard-mounted boundary condition by assuming infinite values of the spring's stiffness and a free-free boundary condition where these are set to zero. The suspension systems were modelled as a combination of five Degrees of Freedom (DoFs), as depicted in Fig. 2. These included:

i. two combinations of linear springs and dashpots, each in one of the two radial translational DoFs ( $\mathrm{x}$ and $\mathrm{y}$ ) 
ii. two pairs of torsional springs and dashpots, each in one of the two radial rotational DoFs $(\theta$ and $\varphi)$

iii. a pair of linear spring and dashpot in the axial translational DoF (z)

Due to axisymmetry, the spring and dashpot values, in the radial translational DoFs and in the radial rotational DoFs, were assumed identical, respectively. The generalised Lagrangian coordinates in the RWA model are ten: $x_{w}, y_{w}, z_{w}, \theta_{w}, \varphi_{w}, x_{b}, y_{b}, z_{b}, \theta_{b}$ and $\varphi_{b}$ contained in the vector $\boldsymbol{q}_{w a}$. The angular rotation w.r.t. z-axis, $\psi_{w}$ and $\psi_{b}$, are not considered due to the assumption of flywheel steady speed rotation and, consequently, domination over angular speed perturbation in the torque DoF.

The mass imbalance can be represented as a point mass $m$, placed at radius $r$ on the flywheel and distance $h$ from the flywheel CoM. This created radial forces and moments when the flywheel spins which amplitudes are function of the radial distance and angular speed. Although the model captures the RWA structural modes including the gyroscopic effects, fundamental harmonics and their amplifications, it does not consider either suband super-harmonics (due to motor bearing imperfections [36]) or broadband noise. The excitation vector, representing the disturbances derived from the flywheel mass imbalance, motor bearing imperfections and broadband noise, can be described superimposing sub- and high- harmonics and broadband noise with the fundamental harmonics [26].

In order to derive the system EoMs in their linearised form, the kinetic energy, the potential energy and the work done were computed following a similar process to that described in [33]. The kinetic energy was defined as the sum of the kinetic energy associated with a balanced flywheel (no mass 
imbalance) and that given by the mass imbalance. The potential energy was derived by applying virtual displacements at the flywheel and wheel-base CoMs and calculating the forces and moments due to the elastic reaction of the suspension systems. Similarly, the work done was calculated by evaluating the viscous forces and moments generated by the dashpots when virtual displacements are applied. The energies and the work done were subsequently implemented in the Lagrange-Euler equation [35]. In the simplification and linearisation processes, small displacements and angles were considered thus to neglect second order terms. Moreover, the flywheel angular speed (about the axial axis) was assumed significantly larger than the angular velocities about the radial axes hence ignoring the second order effects related to the latter.

Finally, the linearised EoMs of the wheel assembly in its matrix form can be expressed as:

$$
\mathbf{M}_{w a} \ddot{\mathbf{q}}_{w a}+\left(\mathbf{C}_{w a}+\mathbf{G}_{w a}\right) \dot{\mathbf{q}}_{w a}+\mathbf{K}_{w a} \mathbf{q}_{w a}=\mathbf{f}_{w a}
$$

where $\mathbf{q}_{w a}$ and $\mathbf{f}_{w a}$ are the vector of the generalised Lagrangian coordinates and the excitation vector, respectively. Matrix $\mathbf{G}_{w a}$ contains terms reproducing the gyroscopic effect. Furthermore, in both stiffness, $\mathbf{K}_{w a}$, and damping, $\mathbf{C}_{w a}$, matrices non-zero off-diagonal elements, indicative of the coupled motion of the radial translational and rotational modes, are considered. The expanded matrices and vectors are reported in Appendix A.

\subsection{Dynamic Coupling Theory}

With the aim to define the impact of the disturbances produced by a microvibration source, two aspects have to be taken into account: static 
and dynamic effect. The former indicates the effects of the source's inertia properties on the dynamics of the supporting structure (i.e. satellite). The latter, on the other hand, represents the effect of the source when this is in operation; for instance, the mass imbalance of a flywheel or the mechanical noise generated by imperfections in the motor bearings. The static effect can be evaluated either using a detailed FE model of the source (although rotary mechanisms $\mathrm{FE}$ models are quite challenging to be reproduced) or using experimental results, as proposed in [31]. Concerning the dynamic influence, the simplest and most common approach is to process the data derived from a hard-mounted configuration test campaign.

The final goal is therefore, to combine both the static and the dynamic effects to generate a single and unique equivalent input force. This can be subsequently used in coupled dynamics analysis as the driving force to be applied at the source in order to move the source itself. In fact, the forces and moments $\mathbf{f}_{C}$ that are actually transmitted at the interface between the source and the supporting structure can be described as function of:

i. dynamic characteristics (inertia, stiffness and damping) of the source, $\mathbf{D}_{w a}$

ii. dynamic characteristics (inertia, stiffness and damping) of the supporting structure, $\mathbf{D}_{s s}$

iii. the driving force used to move the source (i.e. forces and moments measured in the hard-mounted boundary condition $\mathbf{f}_{B}$ )

$$
\mathbf{f}_{C}=\mathbf{f}_{B}-\mathbf{D}_{w a} \ddot{\mathbf{x}}_{C}
$$

where $\ddot{\mathbf{x}}_{C}$ is the vector of the coupled acceleration at the interface. The mainstream idea in this study is that the source and the satellite are connected 
through a single interface node with six DoFs. Consequently, $\mathbf{f}_{C}, \mathbf{f}_{B}$, and $\ddot{\mathbf{x}}_{C}$ are $6 x 1$ frequency dependent vectors, and $\mathbf{D}_{w a}$ is a $6 \times 6$ matrix. The acceleration vector $\ddot{\mathbf{x}}_{C}$ can be determined if the supporting structure response at the interface node point (i.e. dynamic mass of the supporting structure $\mathbf{D}_{s s}$ ) is provided:

$$
\ddot{\mathbf{x}}_{C}=\mathrm{D}_{s s}^{-1} \mathbf{f}_{C}
$$

Substituting Eq.(3) into Eq.(2), Eq.(2) can be re-written as:

$$
\mathbf{f}_{C}=\mathbf{f}_{B}-\mathbf{D}_{w a} \mathbf{D}_{s s}^{-1} \mathbf{f}_{C}
$$

which can be re-arranged for $\mathbf{f}_{C}$ to obtain:

$$
\mathbf{f}_{C}=\left(\mathbf{I}-\mathbf{D}_{w a} \mathbf{D}_{s s}^{-1}\right)^{-1} \mathbf{f}_{B}
$$

where $\mathbf{I}$ is a $6 \mathrm{x} 6$ unit matrix. The difference between the forces measured in the hard-mounted configuration and those derived when the source is physically assembled with its supporting structure is clearly visible. Eq.(5) can also be reformulated in terms of Power Spectral Density (PSD) which shows directly the frequency content of the signals and their relative strength in each DoF. This is also a common and industry accepted format to present this kind of data. The $6 \times 1$ force vectors $\mathbf{f}_{C}$ and $\mathbf{f}_{B}$ become $6 \times 6$ matrices, $\Phi_{C}$ and $\Phi_{B}$, respectively, where the off-diagonal terms supply the cross-correlation knowledge. The term in bracket can be seen as the transfer function between $\mathbf{f}_{B}$ and $\mathbf{f}_{C}$. When dealing with PSD entities, the Hermitian of the matrix representing the transfer function has to be calculated. The output coupled force $\Phi_{C}$ can be finally expressed as:

$$
\Phi_{C}=\left(\mathbf{I}-\mathbf{D}_{w a} \mathbf{D}_{s s}^{-1}\right)^{-1} \Phi_{B}\left(\mathbf{I}-\mathbf{D}_{w a} \mathbf{D}_{s s}^{-1}\right)^{-H}
$$


The PSD output at any location on the satellite structure, $\Phi_{\text {out }}$, can subsequently be obtained multiplying the $\Phi_{C}$ by an opportune transfer function matrix which links the interface node point to the output location point on the satellite structure, $\mathbf{T F}_{C-\text { out }}$, thus to obtain:

$$
\Phi_{\text {out }}=\mathbf{T F}_{C-\text { out }}\left(\mathbf{I}-\mathbf{D}_{w a} \mathbf{D}_{s s}^{-1}\right)^{-1} \Phi_{B}\left(\mathbf{I}-\mathbf{D}_{w a} \mathbf{D}_{s s}^{-1}\right)^{-H} \mathbf{T F}_{C-\text { out }}^{H}
$$

The predictions obtained using Eq. (6) and Eq. (7) can be quantitatively compared to the experimental results in terms of FRAC. This evaluates each DoF based on the frequency response comparison of the analytical and experimental derived functions [32]. The FRAC can assume values comprised between 0 and 1, where 1 indicates perfect correlation. The FRAC is expressed as:

$$
F R A C=\frac{\left(\sum_{i}\left\{\Phi_{\text {out }_{\text {test }}}\right\}\left\{\Phi_{\text {out }, F E M_{i}}\right\}^{*}\right)^{2}}{\left(\sum_{i}\left\{\Phi_{\text {out }_{\text {test }}}\right\}\left\{\Phi_{\text {out }_{\text {test }}}\right\}^{*}\right)\left(\sum_{i}\left\{\Phi_{\text {out }, F E M_{i}}\right\}\left\{\Phi_{\text {out }, F E M_{i}}\right\}^{*}\right)}
$$

where $*$ represents conjugate values. Note that no stretching and no shifting has been performed in this study.

\subsection{Dynamic Mass Modelling}

In general, the dynamic mass can be expressed as a fully populated $6 \mathrm{x} 6$ matrix. Previous studies in $[20,37,38]$ have shown that the diagonal elements are the most influential. In addition, the four cross-term dynamic mass elements $D_{w a 15}, D_{w a 24}, D_{w a 42}, D_{w a 51}$ are also significant. Furthermore, due to the gyroscopic effect, the off-diagonal elements correlating the translational DoFs and the rotational DoFs, commonly neglected, are also important hence must be considered [39]. Being the dynamic mass matrix symmetric about its diagonal and due to RWA axisymmetry, the following assumptions can be made: 
i. no coupled effects between the axial and radial modes are considered so that elements $D_{w a i 3}$ for $i \neq 3, D_{w a 3 j}$ for $j \neq 3, D_{w a i 6}$ for $i \neq 6$ and $D_{w a 6 j}$ for $j \neq 6$, are equal to zero

ii. $D_{w a 11}=D_{w a 22}$

iii. $D_{w a 44}=D_{w a 55}$

iv. $D_{w a 15}=D_{w a 51}$

v. $D_{w a 24}=D_{w a 42}$

vi. $D_{w a 24}=-D_{w a 15}$

vii. $D_{w a 21}=-D_{w a 12}$

viii. $D_{w a 14}=D_{w 25}$

ix. $D_{w a 41}=D_{w 52}=-D_{w a 14}=D_{w a 25}$

X. $D_{w a 54}=-D_{w a 45}$

Thereby, the dynamic mass matrix can be written as:

$$
\mathbf{D}_{w a}=\left[\begin{array}{cccccc}
\hline D_{w a 11} & D_{w a 12} & 0 & D_{w a 14} & -D_{w a 24} & 0 \\
-D_{w a 12} & D_{w a 11} & 0 & D_{w a 24} & D_{w a 14} & 0 \\
0 & 0 & D_{w a 33} & 0 & 0 & 0 \\
-D_{w a 14} & D_{w a 24} & 0 & D_{w a 44} & D_{w a 45} & 0 \\
-D_{w a 24} & -D_{w a 14} & 0 & -D_{w a 45} & D_{w a 44} & 0 \\
0 & 0 & 0 & 0 & 0 & D_{w a 66}
\end{array}\right]
$$

Note the number of elements defining the RWA dynamic mass matrix is dramatically reduced from 36 to only 8 elements, boxed in Eq.(9). In order to analytically and/or experimentally retrieve the dynamic mass of a RWA, direct measurements of the wheel accelerance matrix [31, 40] are generally carried out. 
(a)

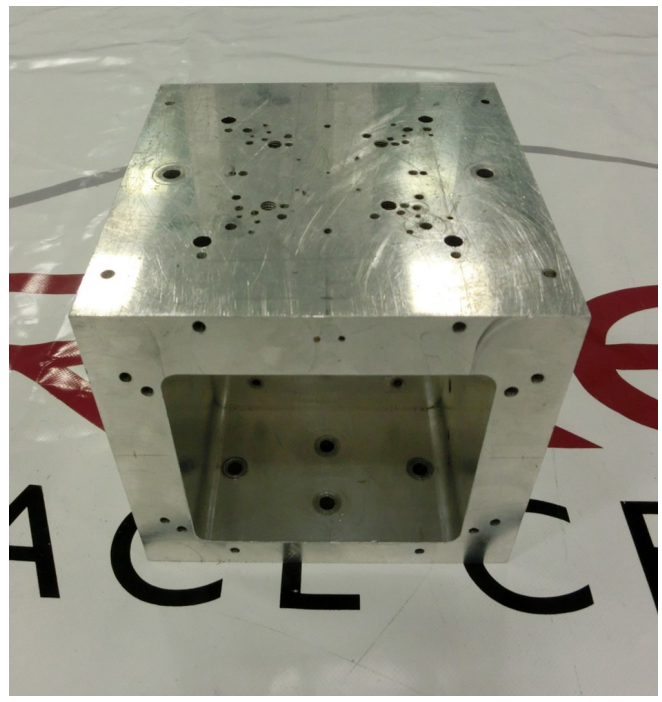

(b)

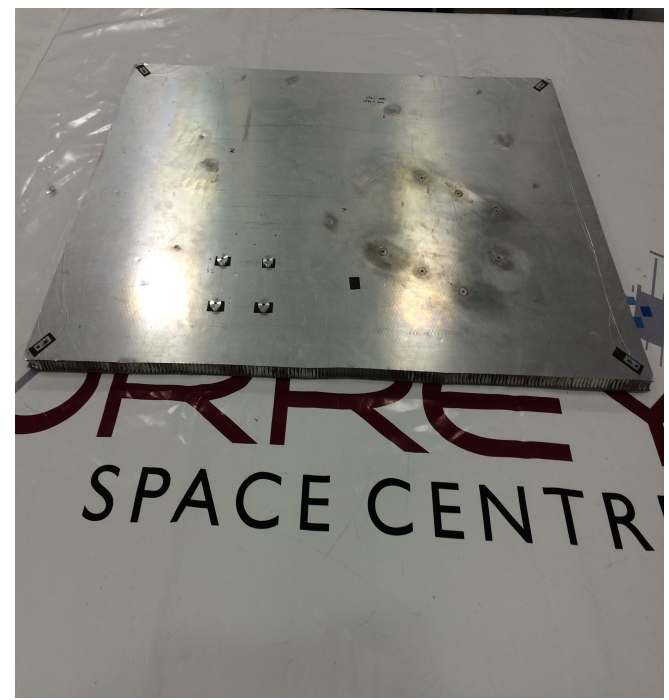

Fig. 3 .

Supporting structures used during coupled test campaign: (a) stiff support and (b) honeycomb panel. The stiff support is a solid block made of Aluminium whereas the panel is a sandwich structure.

\section{Experimental Tests}

A preliminary test campaign was conducted to assess the loads, due to mass imbalance and local motor bearing imperfections, generated by the RWA when hard-mounted on an isolated and rigid platform. Subsequently, the coupled dynamics when the RWA is assembled with a stiff support (see Fig. 3(a)) were measured, in terms of coupled forces at the RWA-structure interface and accelerations at several locations on the structure, and the outcomes used to validate the RWA model and the equations described in section 2. Finally, the RWA was connected to a more representative spacecraft structure, shown in Fig. 3(b), and the interface loads and accelerations 
(a)

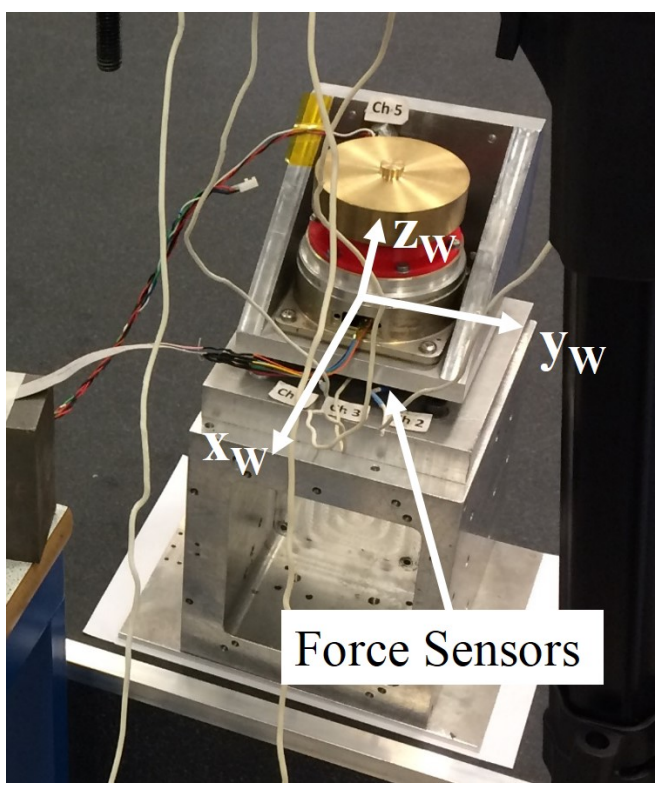

(b)

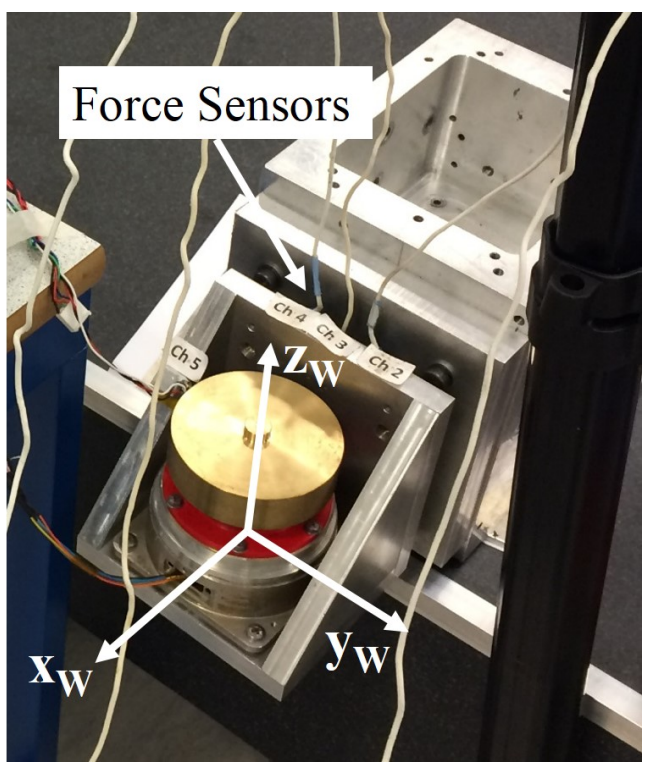

Fig. 4 .

RWA hard-mounted test setup: (a) RWA z-axis and (b) RWA x-axis.

of the flexible supporting structure quantified by means of uni-axial force transducers and accelerometers. The experimental tests have been carried out in the Surrey Space Centre facility and, in order to minimise the level of noise, they have been run between $12 \mathrm{am}$ and $6 \mathrm{am}$ significantly restricting the noise due to normal activities inside and outside the building (e.g. traffic, walking, etc.).

\subsection{Hard-mounted Configuration}

The loads generated by the RWA were measured using the platform developed in [40]. A range of speed from 600 to $4800 \mathrm{rpm}$ with a $60 \mathrm{rpm}$ step increase was considered and the acquisition time was set to 8 seconds leading to a total of 71 available measurements and 28 minutes of recording. Due 
to the use of uni-axial force transducers, three test setups were needed for the full characterisation of the RWA-induced loads. Fig. 4 illustrates the test configuration for measurements in the wheel z-axis and x-axis. Measurements along the wheel y-axis were obtained by rotating the RWA x-axis test configuration by 90 degrees clockwise. Given the relative position of the three uni-axial force transducers used in the experiments, it was also possible to derive the moments at the RWA-platform interface. In addition, with the aid of a Kapton tape taped onto the flywheel and of an optical sensor, the signals were firstly synchronised and subsequently post-processed.

The results from the experiments are shown as spectral maps in Fig. 5. These display the forces and moments at the RWA-platform interface and a prevalence of the fundamental harmonic with respect to sub and higher harmonics can be observed. Furthermore, they provide a qualitative overview of the results giving an indication of the relationship between the secondary harmonics and the structural modes of the system, the latter expressed in terms of Campbell diagram and plotted as black solid lines. These serve to show how the interaction of the harmonics with the RWA structural modes leads to amplification in the RWA response. For instance, the amplification occurring at $117 \mathrm{~Hz}$ in Fig. 5(b) due to interference of the second harmonic and higher harmonics with the axial structural mode of the RWA.

\subsection{RWA-stiff-support Coupled Configuration}

The RWA was secured to a stiff Aluminium structure whose mass is ten times bigger than the RWA's mass. The system was then hung using elastic cords to reproduce a free-free boundary condition. A set of four uni-axial force sensors was placed at the interface between the RWA and the stiff 
(a)

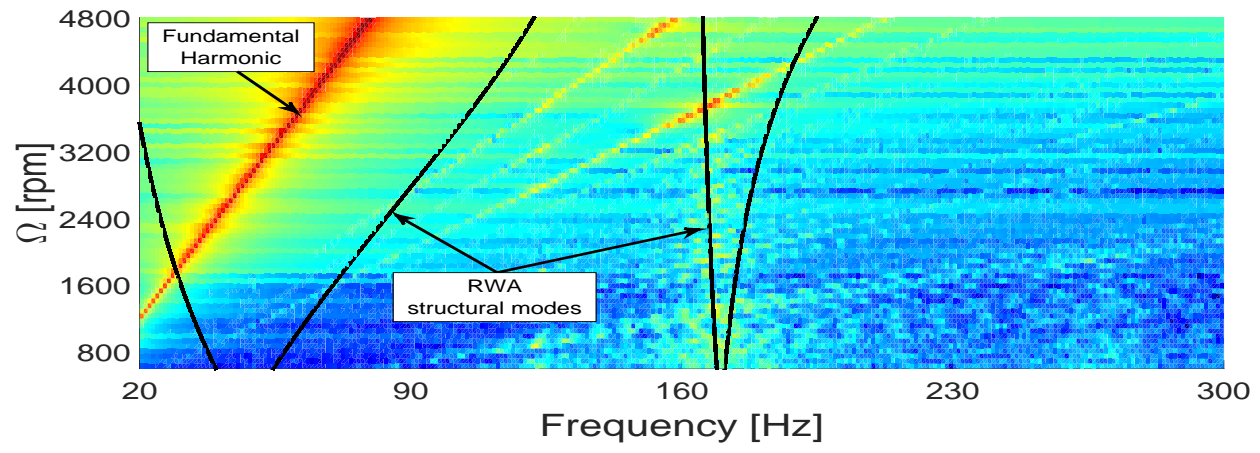

(b)

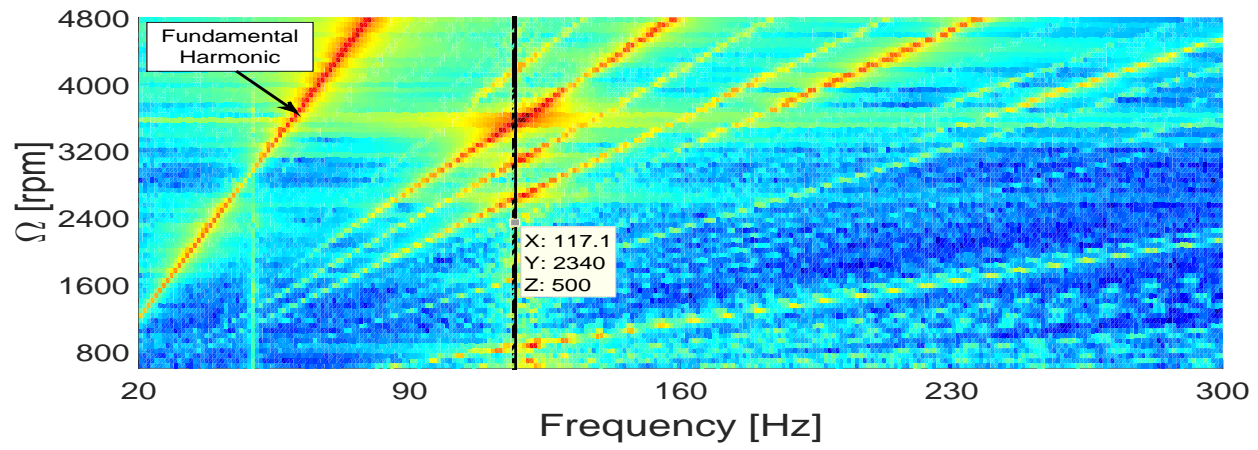

(c)

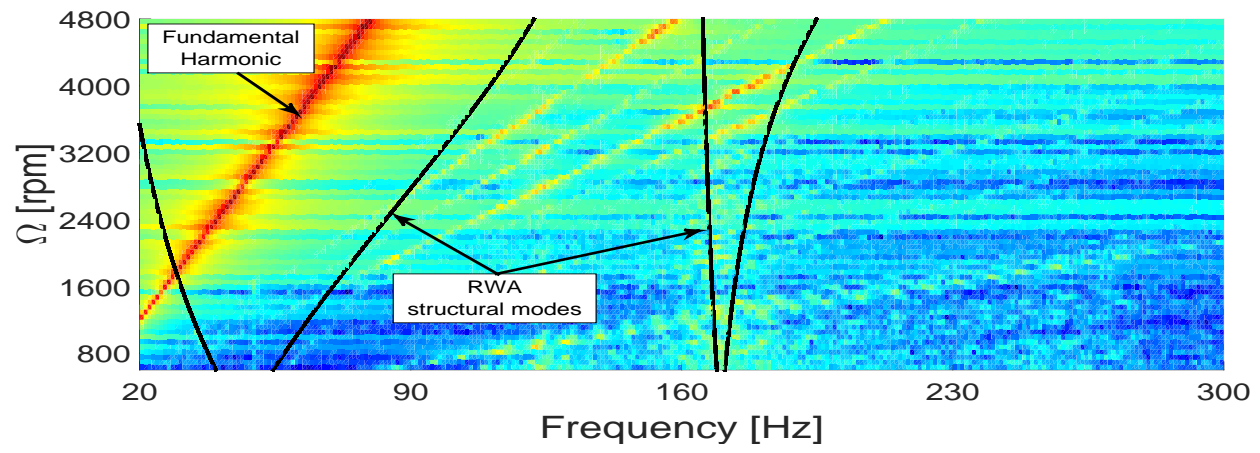

Fig. 5.

Spectral maps of loads generated in the hard-mounted configuration superimposed to the RWA speed dependent structural modes expressed as Campbell diagram (black solid lines):

(a) $\Phi_{B 11}$; (b) $\Phi_{B 33}$ and (c) $\Phi_{B 55}$. 

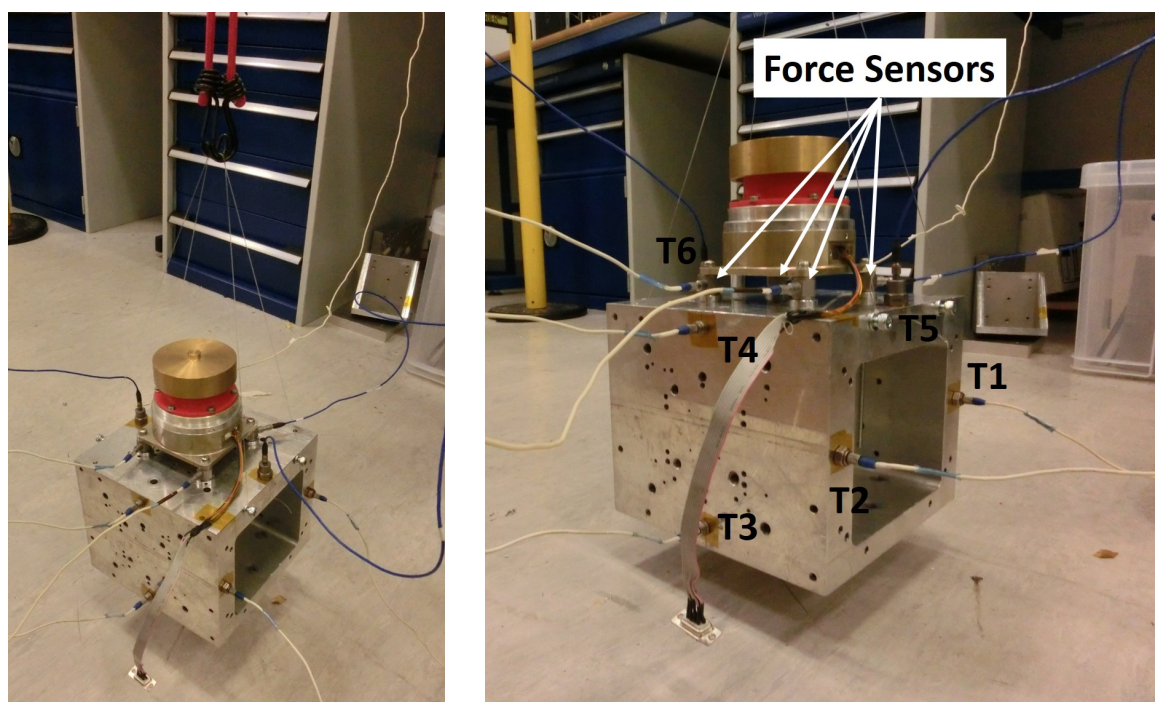

Fig. 6 .

Coupled RWA-stiff-support test setup.

support to measure the coupled loads exchanged by the two bodies. Furthermore, six accelerometers were positioned on the stiff support to evaluate its accelerations due to the RWA-induced excitation. A combination of the six responses allowed to fully characterise the motion of the stiff support in all the DoFs (three translations and three rotations). The test setup is shown in Fig. 6. The RWA was spun from 1200 to $4800 \mathrm{rpm}$ with a $300 \mathrm{rpm}$ step increase and the output data are represented as PSD waterfall plots in Fig. 7.

Due to the rigidity of the supporting platform, the system displays a similar behaviour to that observed in the hard-mounted configuration. For instance, the interaction between higher harmonics and the RWA-stiff-support speed dependent structural modes is well depicted in Fig. 7. Amplifications occurring when the harmonic frequency matches the corresponding system 
(a)

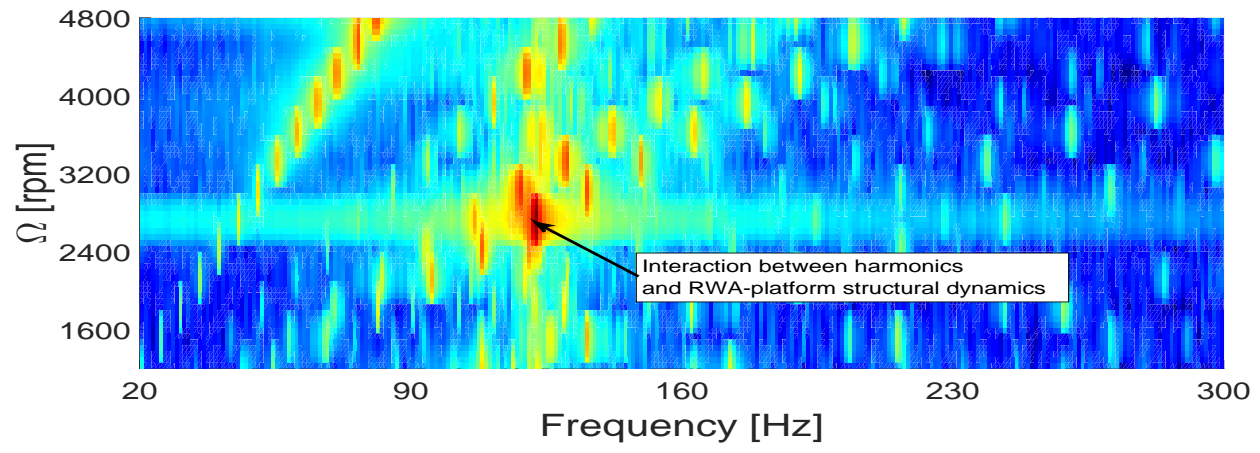

(b)

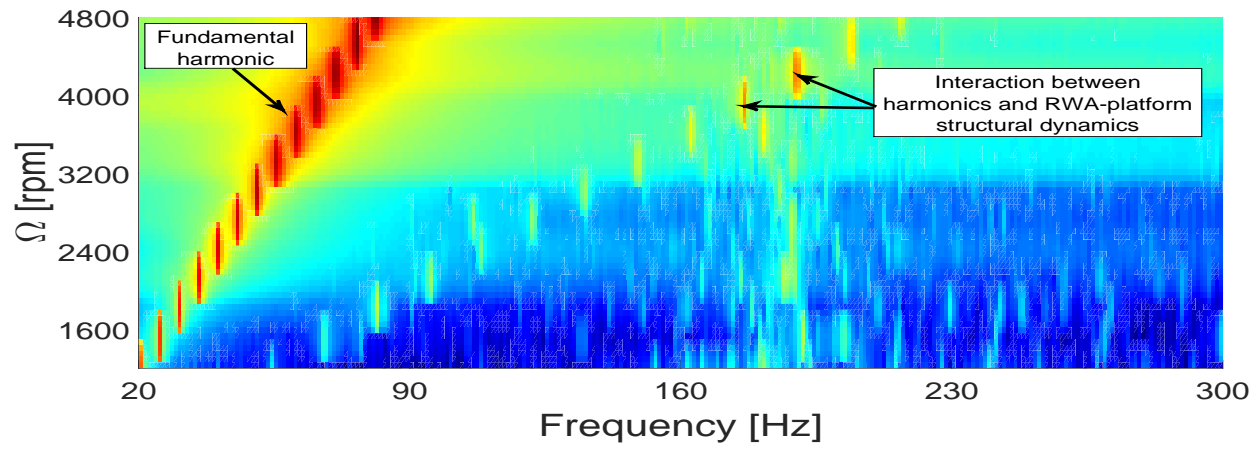

(c)

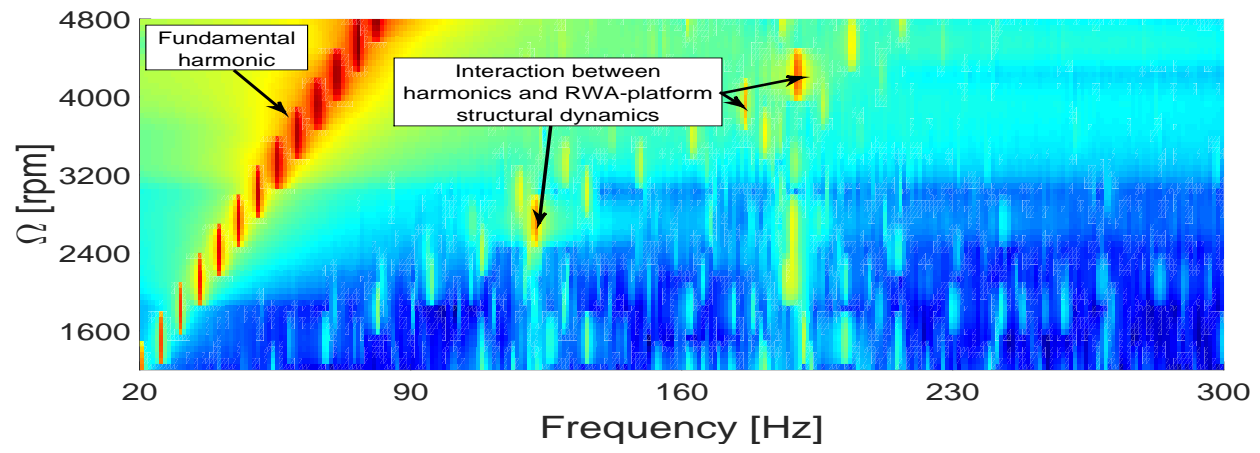

Fig. 7.

Spectral maps of the coupled RWA-stiff-support experimental data: (a) axial force; (b) moment and (c) accelerometer T3. Amplifications due to the interaction between harmonics and the system structural modes are also highlighted. 
structural mode are highlighted in Fig. 7 for frequencies equal to about 120 $\mathrm{Hz}$ and $190 \mathrm{~Hz}$.

\subsection{RWA-panel Coupled Configuration}

A sandwich panel with Aluminium skin (0.9 mm thick) and Aluminium honeycomb core (18 mm thick) was selected as representative of a flexible spacecraft interface. The RWA was connected to the panel by means of 4 force sensors $(100 \mathrm{mV} / \mathrm{N}$ sensitivity). These were used to measure the coupled forces along the RWA z-axis at the RWA-to-panel interface. A set of 3 accelerometers $(1000 \mathrm{mV} / \mathrm{g}$ sensitivity) suitable for microvibration analysis was installed on different locations of the panel with the purpose to evaluate the structure response to the RWA-induced disturbances. Finally, in order to reproduced a free-free boundary condition, the system was lifted from the ground using a crane and elastic cords. The free-free condition was such that the rigid body natural frequencies of the system were lower than $1 \mathrm{~Hz}$. In Fig. 8, the test configuration is illustrated, also showing the location of the accelerometers.

The RWA flywheel was operated using an opportunely designed motor

driver in a range of speed spacing from $600 \mathrm{rpm}$ to $4800 \mathrm{rpm}$. Increments of $60 \mathrm{rpm}$ were applied allowing a resolution in the speed domain of $1 \mathrm{~Hz}$.

The data were acquired in the time domain and subsequently processed and reformulated in the frequency domain in terms of PSD. The outcomes of this analysis are graphed in Fig. 9 as spectral maps. The interaction between the RWA-induced disturbances and the RWA-panel structural modes is clearly present and amplifications occurring at frequencies for whose the harmonics frequency and the system resonance match can be observed. For 

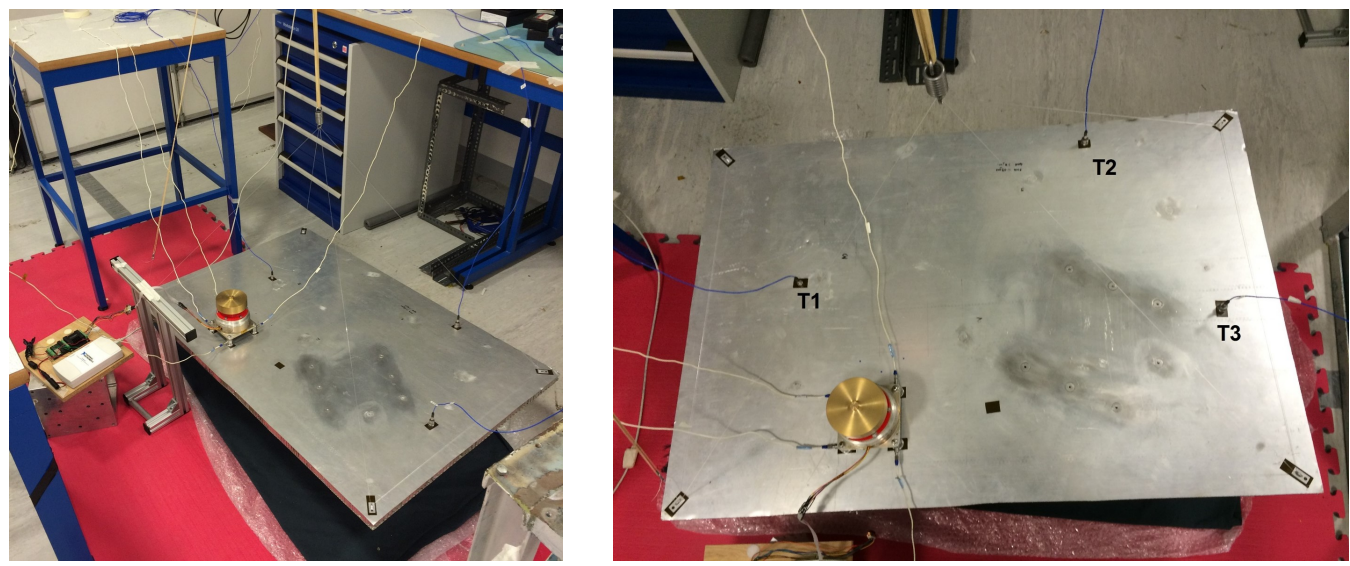

Fig. 8 .

Coupled RWA-panel test setup.

instance, increases in the system response amplitude are experienced at frequencies equal to $100 \mathrm{~Hz}, 150 \mathrm{~Hz}, 230 \mathrm{~Hz}$ and $280 \mathrm{~Hz}$ as depicted in Figs. 9(a) and 9(c). Further amplifications are also registered at $160 \mathrm{~Hz}$, as shown in Fig. 9(b). It must be remarked that when dealing with coupled dynamics analysis, the coupling between the RWA internal dynamics and the supporting structure structural dynamics is significant.

\section{Coupled Dynamics Analysis}

The static frequency only dependent RWA dynamic mass was initially retrieved experimentally using the method described in [31]. This allowed an accurate FE model of the RWA (Fig. 10(a)) to be built and further expanded to include the gyroscopic effect. This was used to compute the RWA dynamic in both the frequency and speed ranges of interest.

In this section, the frequency and speed dependent RWA dynamic mass at the mounting point location in both static and in-operation conditions are 
(a)

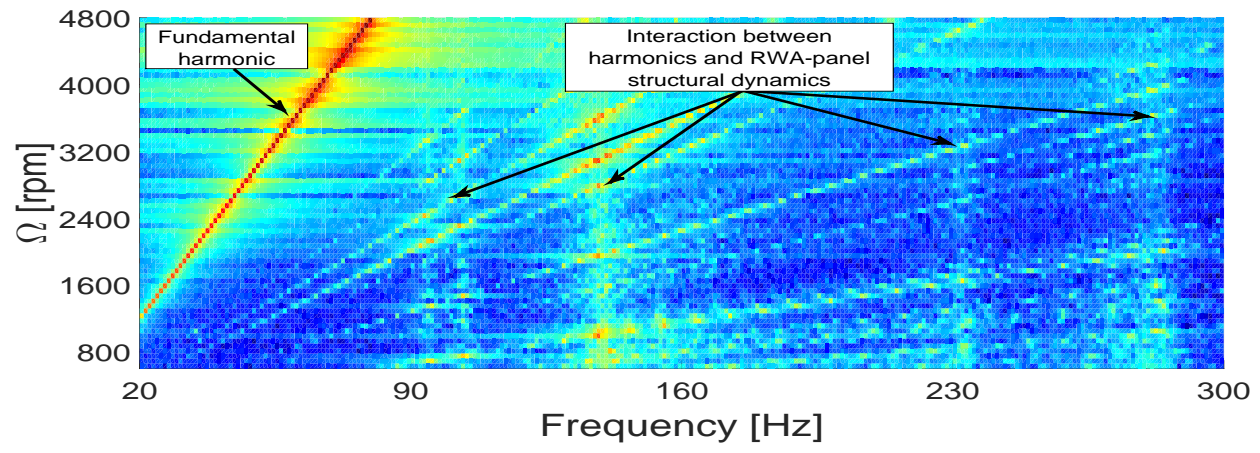

(b)

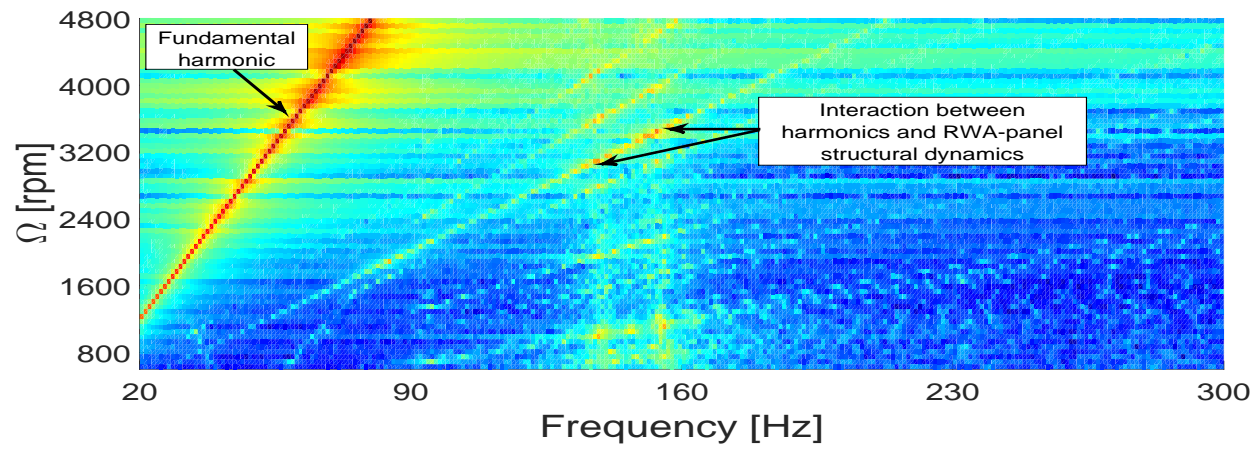

(c)

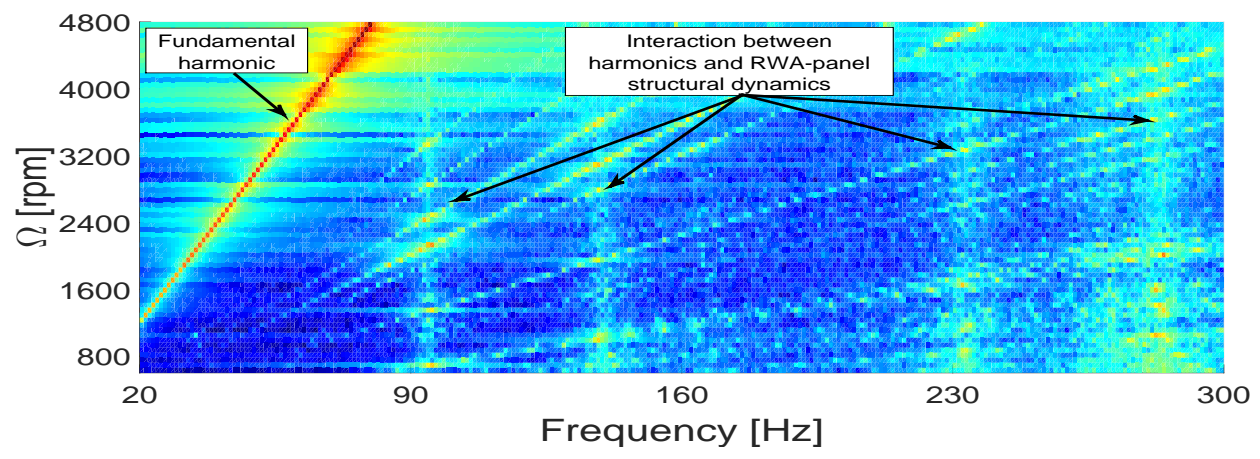

Fig. 9.

Spectral maps of the coupled RWA-panel experimental data: (a) axial force; (b) moment and (c) accelerometer T2. Amplifications due to the interaction between harmonics and the system structural modes are also emphasised. 
(a)

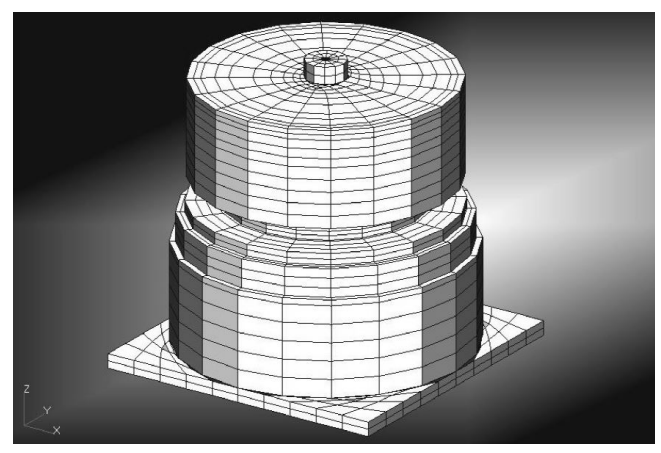

(b)

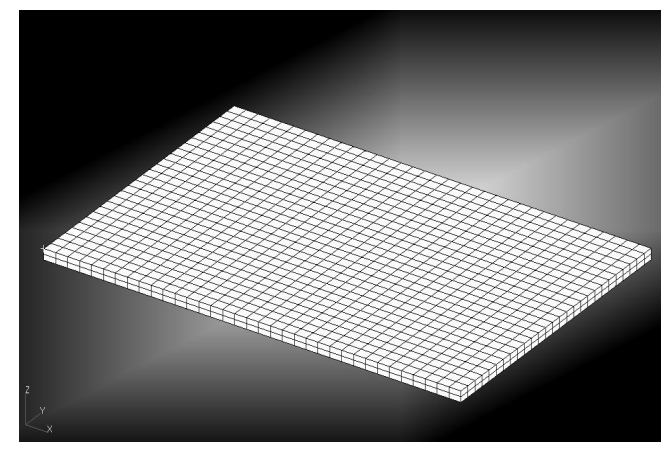

Fig. 10.

FE models: (a) RWA and (b) structural panel.

obtained by means of frequency response analysis in MSC/NASTRAN on the RWA FE model, as depicted in Fig. 10(a). Data processing was performed using a Math tool software (i.e. MATLAB). In addition, the frequency dependent dynamic mass of both the stiff support and the structural panel used for coupled analysis, see Fig. 10(b), are also calculated. The dynamic mass matrices of the RWA, stiff support and structural panel are subsequently implemented with the forces and moments retrieved from hard-mounted experimental tests on the RWA, to estimate the coupled loads transmitted at the interface between the bodies.

\subsection{RWA and Structural Panel Dynamic Mass}

Although the evaluated RWA dynamic mass coefficients displayed good agreement with the experimental results and behaved accordingly, for simplicity, only three elements are shown in this article; they are: $D_{w a 14}, D_{w a 24}$ and $D_{w a 33}$. The structural panel interface point dynamic mass was obtained from its FE model by applying unit forces and moments at one DoF at a 
time; it was then validated against test results described in [41]. Here the panel was suspended using elastic cords as to reproduce a free-free boundary condition. Mini-shakers were then used to excite the six DoFs of the panel at the interface location. The acceleration responses were then post-processed to derive the accelerance matrix of the structure. For ease of legibility, only the element representing the dynamic mass in the axial DoF is reported here as it is the most significant. The analytical responses were simulated in both frequency (20 to $300 \mathrm{~Hz}$ ) and speed (0 to $4800 \mathrm{rpm}$ ) bands for the RWA and in the frequency band only for the supporting platforms. The outcomes are illustrated in Fig. 11 and Fig. 12, respectively. The RWA modes are clearly shown in each plot. Due to the gyroscopic effect and the coupled dynamics of the in-plane DoFs, the system response displays four whirls in Fig. 11(a) and (b): forward and backward whirls of the rotational mode and of the lateral translation mode. These whirls are strongly related to the gyroscopic effect occurring whenever a rotating mechanism displays a conical mode shape. For increasing angular speeds, if the shaft spin and the whirl directions are the same (forward whirl) the gyroscopic effect acts as a stiffener element for the radial rotational mode (at times, also referred to as rocking) of the mechanism hence the resonance frequency of the system grows. On the other hand, if the motion of the whirl is opposite to that of the shaft spin rotation (backward whirl), the gyroscopic effect operates as a softener element thus reducing the stiffness of the mechanism and, therefore, its natural frequencies [42]. Due to the coupled DoF dynamics, for cantilever-configured RWAs, this effect affects both the radial rotational mode and the radial translational mode. $D_{w a 24}$ includes all in-plane structural modes, as expected. Moreover, 
(a)

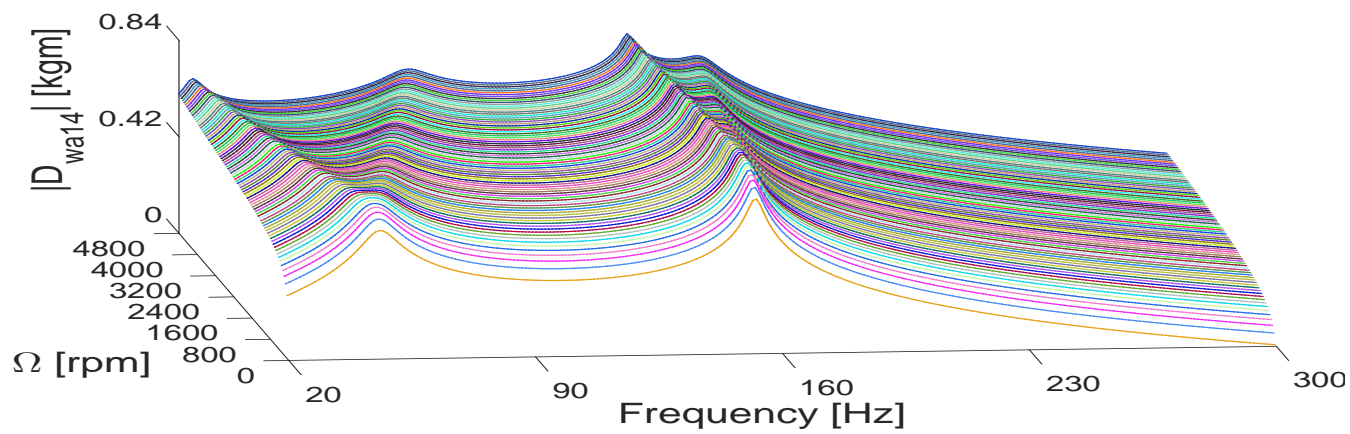

(b)

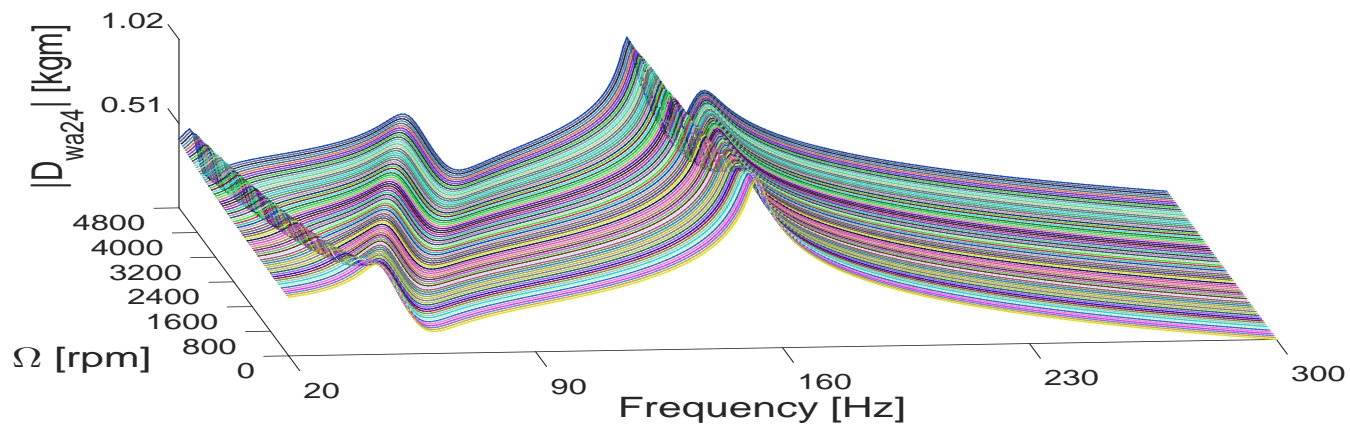

(c)

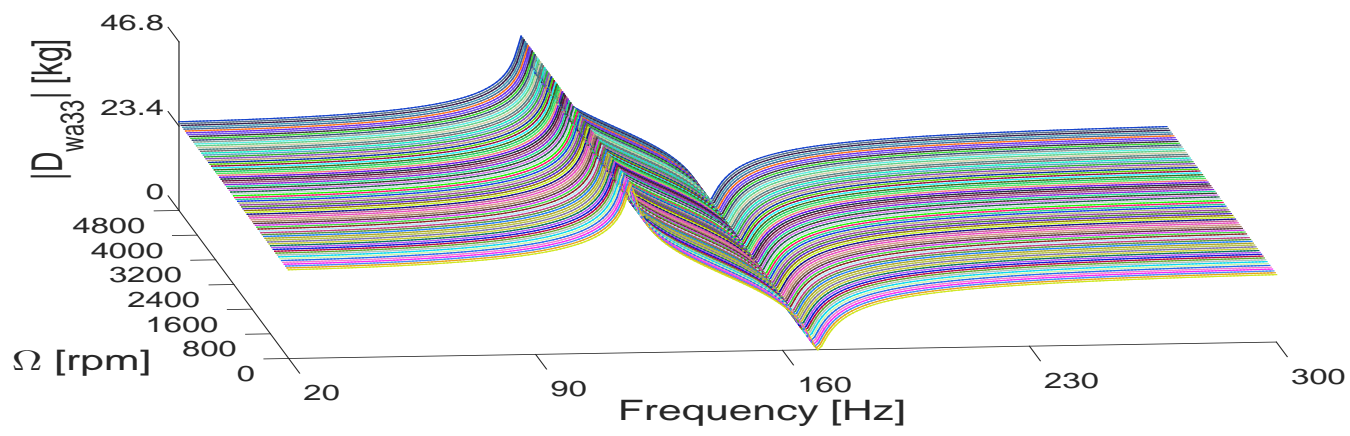

Fig. 11.

Speed and frequency dependent RWA dynamic mass: (a) $D_{\text {wa14 }}$; (b) $D_{\text {wa24 }}$ and (c) $D_{\text {wa33 }}$. 


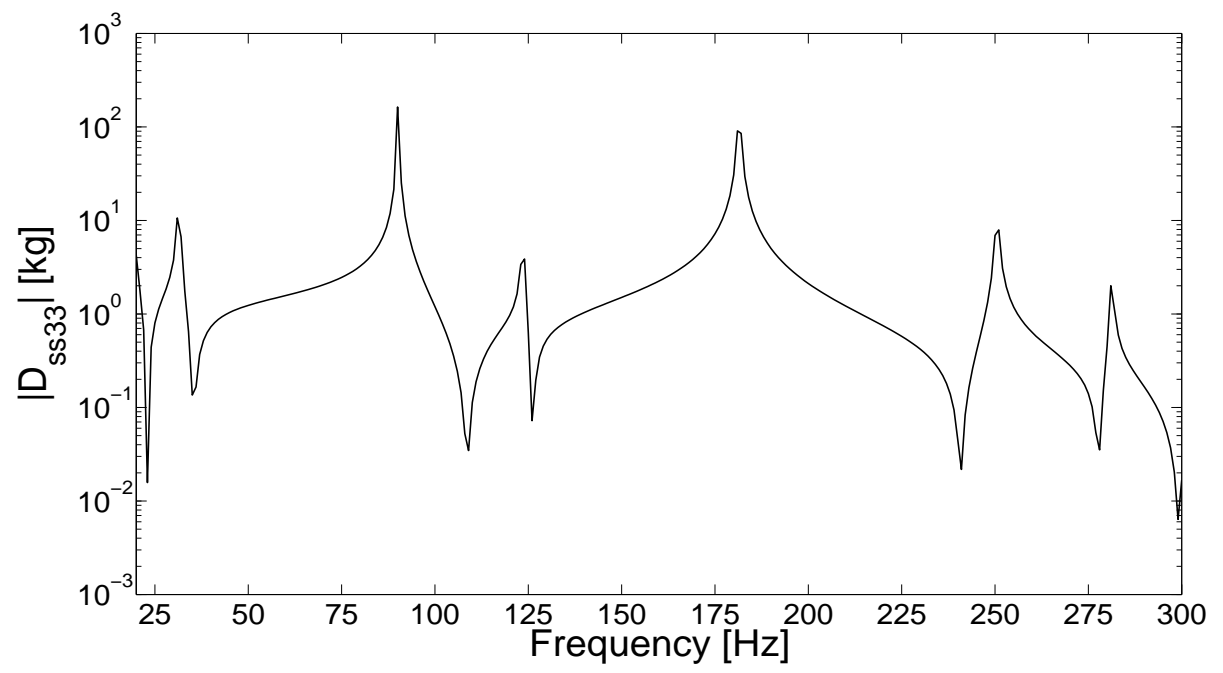

Fig. 12.

Frequency only dependent panel dynamic mass axial DoF element.

the commonly neglected coefficient $D_{\text {wa14 }}$, though null when the RWA is in a zero-speed condition, significantly changes with the spin speed, as a consequence of the gyroscopic effect. The axial mode in Fig. 11(b), in contrast, is decoupled from the in-plane modes and constant throughout the speed range.

\subsection{Coupled Microvibration Analysis}

Figure 13 illustrates the four different cases analysed for both the RWAstiff-support and the RWA-panel systems:

i. source not included in the model and hard-mounted loads directly applied to the supporting structure (e.g. stiff support and panel)

ii. source represented as a lumped mass connected rigidly to the supporting structure (i.e. using rigid elements) 
(a)

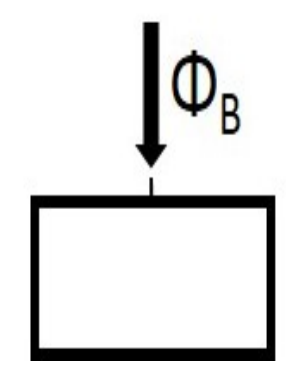

(b)

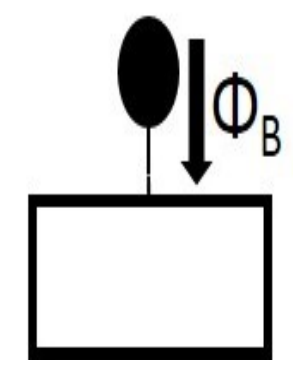

(c)

(d)
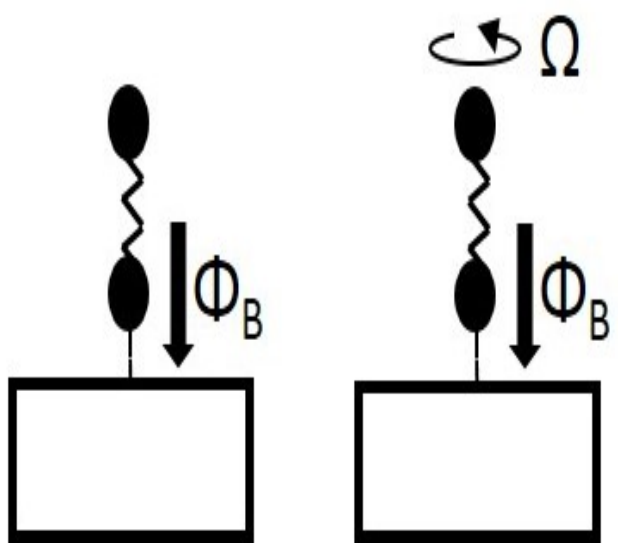

Fig. 13.

Cases analysed: (a) no source; (b) source as lumped mass; (c) source dynamic mass, $D_{w a}(\omega)$ and (d) source dynamic mass including the gyroscopic effect, $D_{w a}(\omega, \Omega)$. $\mathbf{\Phi}_{B}$ represents the PSD of the loads measured in a hard-mounted boundary condition.

iii. source frequency dependent internal dynamics included in the model (the gyroscopic effect is ignored) connected rigidly to the supporting structure (i.e. using rigid elements)

iv. source speed and frequency dependent internal dynamics included in the model (the gyroscopic effect is included) connected rigidly to the supporting structure (i.e. using rigid elements)

The PSD of the hard-mounted loads derived in section 3 were implemented with the analytical dynamic mass of the RWA and the accelerance of either the stiff support or of the structural panel, in Eq. (6) and Eq. (7), to predict the transferred loads and the response of the structures. 
Table 1.

FRAC calculated for the RWA-rigid-platform coupled system responses

\begin{tabular}{lccc}
\hline & Model (a) & Model (c) & Model (d) \\
\hline \hline$\Phi_{C 33}$ & 0.5653 & 0.5807 & 0.5807 \\
$\Phi_{C 44}$ & 0.9980 & 0.9981 & 0.9982 \\
$a_{c u b e, a x}$ & 0.9956 & 0.9957 & 0.9957 \\
\hline
\end{tabular}

The analytical outcomes concerning the coupling between the RWA and the stiff support displayed a good agreement with the experimental data both in terms of interface loads and stiff support response, as shown in Figs. 14 and 15 .

It must be observed that, however, due to its rigidity the stiff support behaves as a lumped mass in the range of frequency analysed (its resonance frequencies are above $1000 \mathrm{~Hz}$ ), hence its internal dynamics did not affect the response. Therefore, the coupled effect between the source and the supporting structure were limited in this case. This led to a simplification of the case-study analysed, for which all the models in Fig. 13 were able to correctly predict the dynamics of the bodies when coupled together. Nevertheless, also in this simplified scenario, the model (d) presented an improved estimate, especially where the internal dynamics of the RWA were important. This can be further demonstrated using the FRAC, as shown in Table 1. The values confirm the good agreement between the analytical results and the test experiment and highlight the superior prediction due to the implementation of the RWA dynamic mass. 
(a)

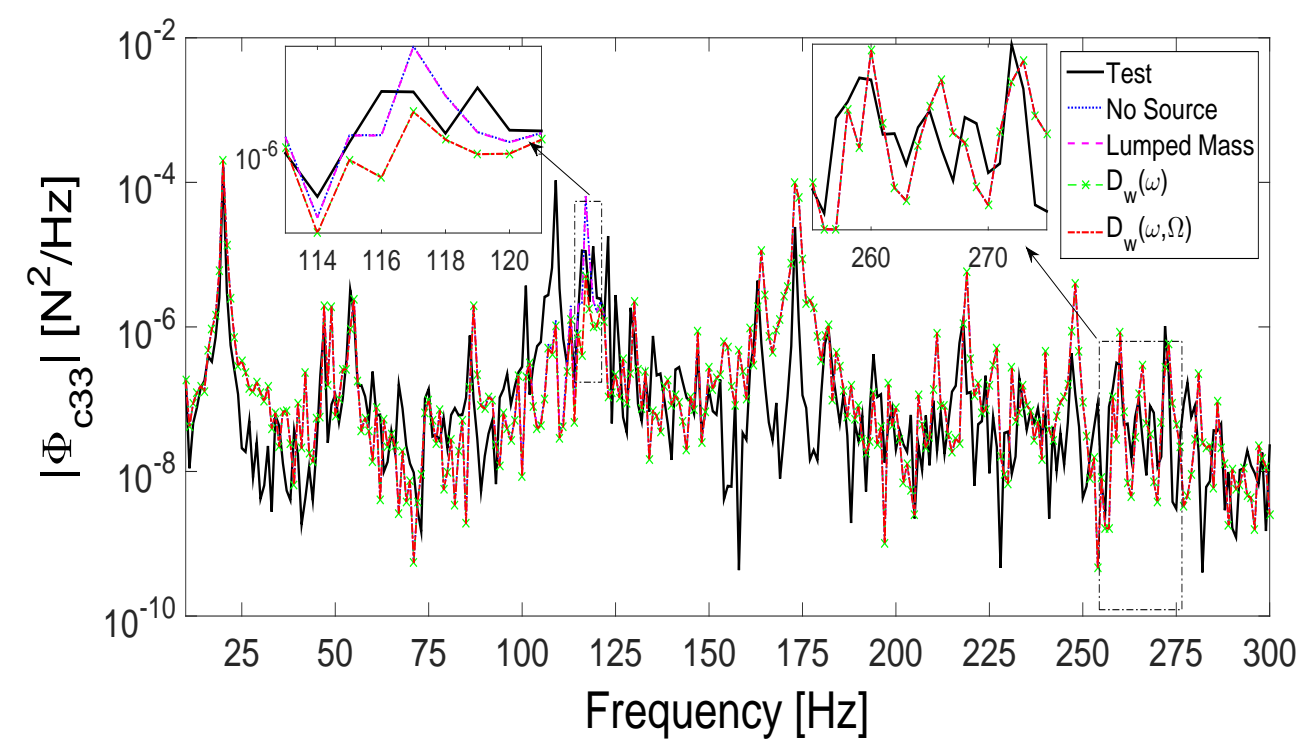

(b)

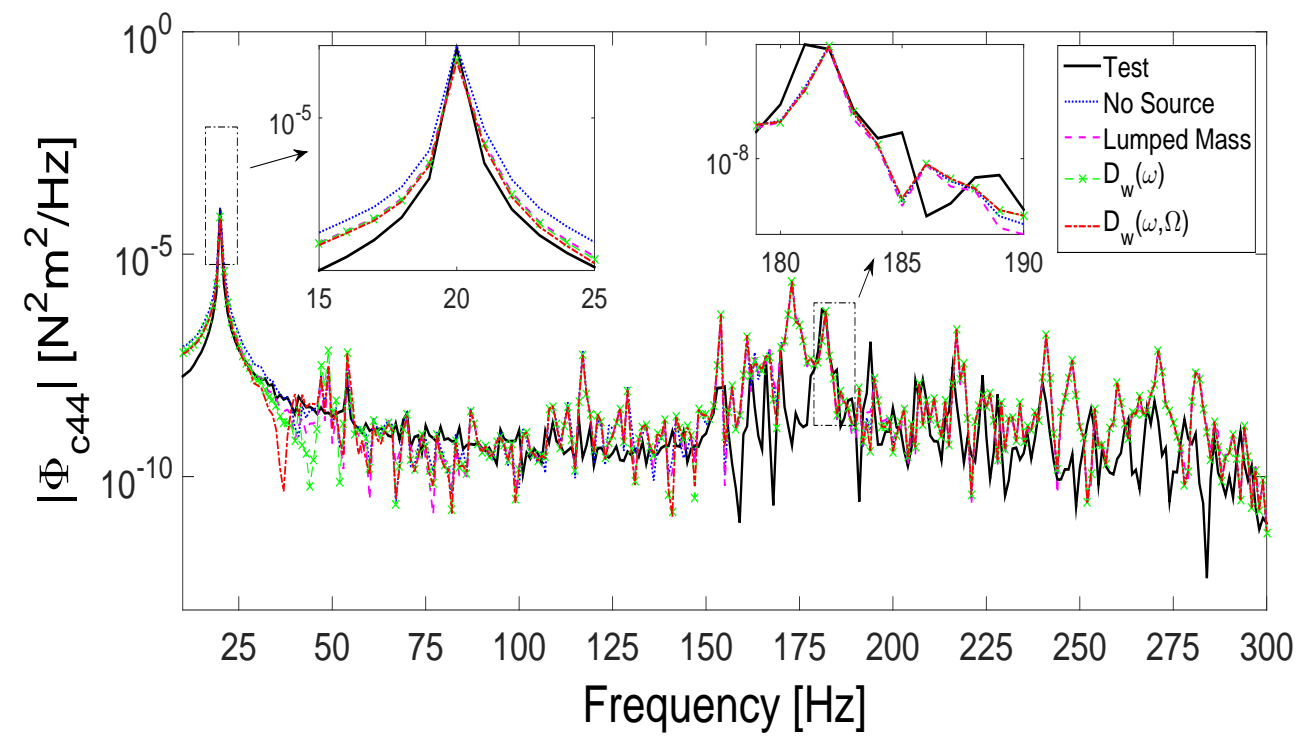

Fig. 14.

Comparison of the interface RWA-stiff platform loads between test results and the four analytical approaches $(\Omega=1200 \mathrm{rpm})$ : (a) $\left|\Phi_{C 33}\right|$ and (b) $\left|\Phi_{C 44}\right|$. 


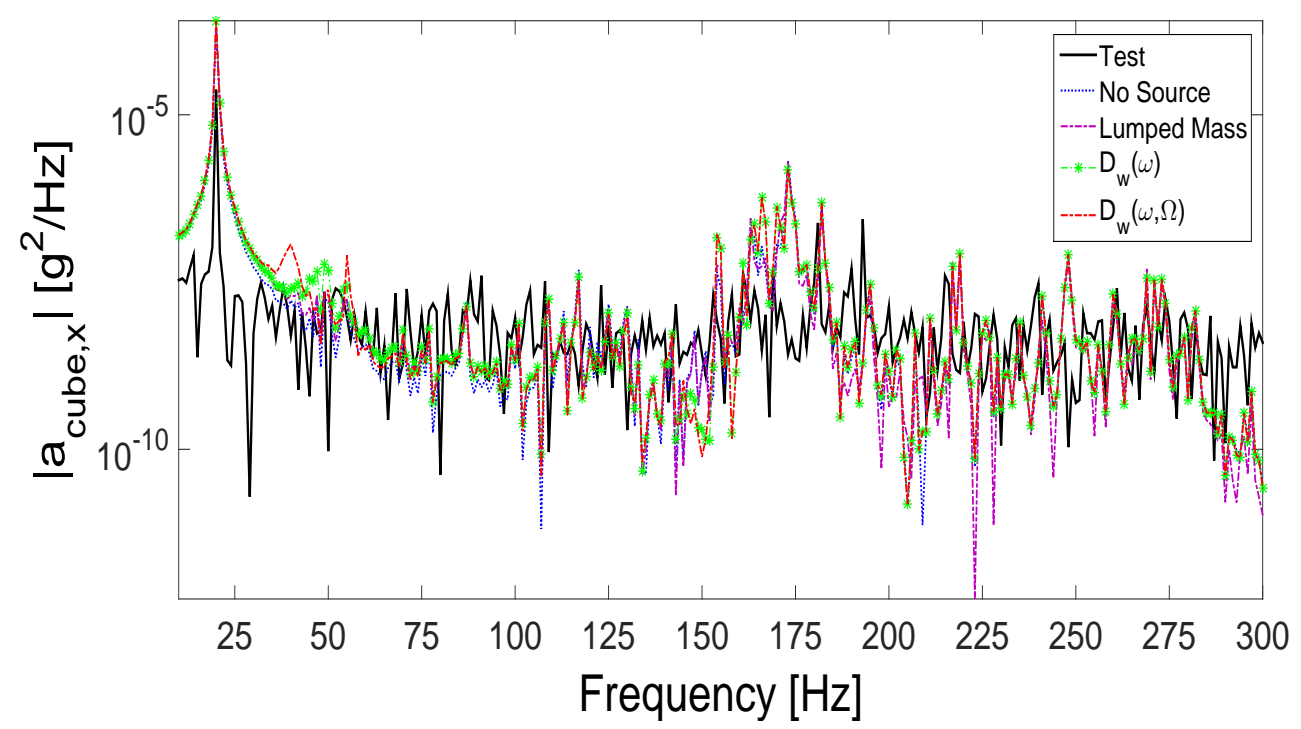

Fig. 15.

Comparison of the acceleration response of the stiff platform between test results and the four analytical approaches $(\Omega=1200 \mathrm{rpm})$.

On the other hand, coupled effects were expected in the RWA-panel coupled configuration due to the flexible behaviour of the structural panel. These can be observed in Figs. 16 and 17, where the experimental results are compared to the analytical estimations.

Resonances due to the interaction between the RWA and the panel internal dynamics are clearly visible. For instance, amplifications can be observed at about $92 \mathrm{~Hz}, 165 \mathrm{~Hz}$ and $215 \mathrm{~Hz}$. In terms of correlation, none of the models in Fig. 13 precisely matched the test results. The hard-mounted microvibrations applied as direct input in model (a) generally overestimated the coupled loads and response, as expected. Moreover, the dynamics of the RWA between $150 \mathrm{~Hz}$ and $175 \mathrm{~Hz}$ were missed. Although improving the response estimate, model (b) failed to simulate the RWA structural modes due 
(a)

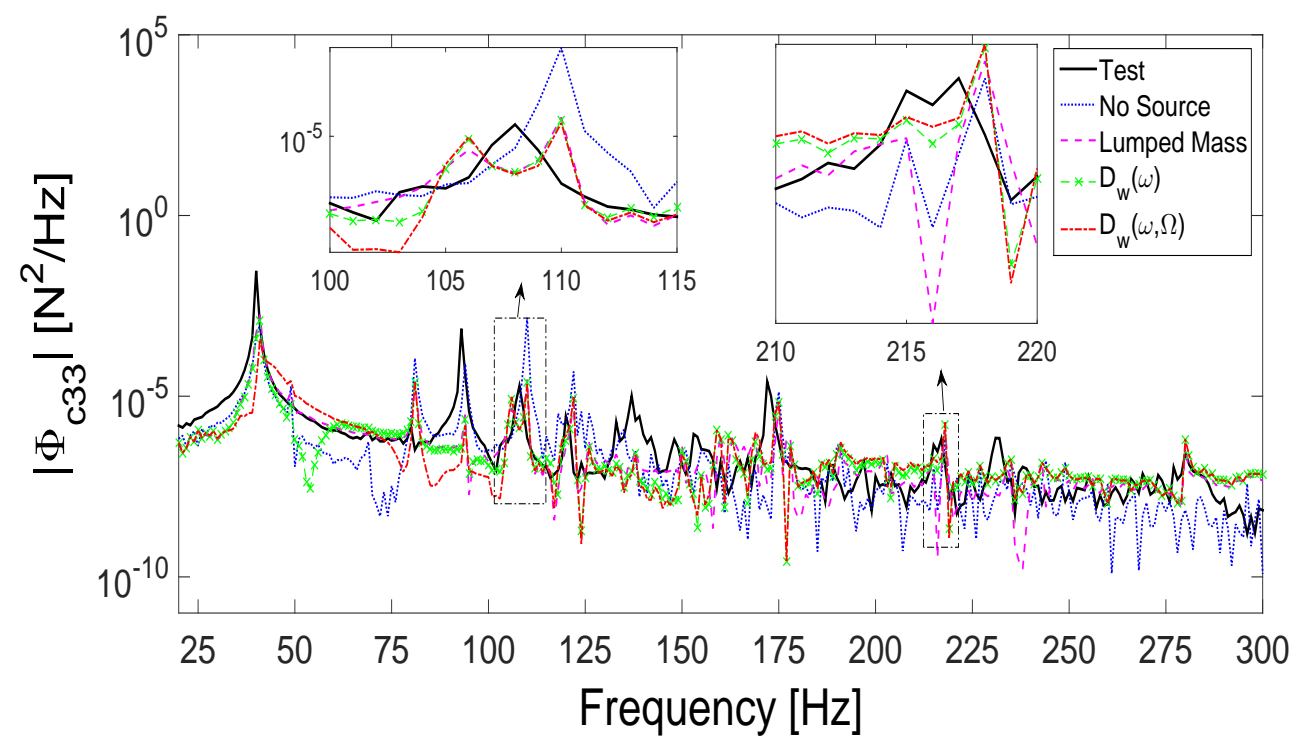

(b)

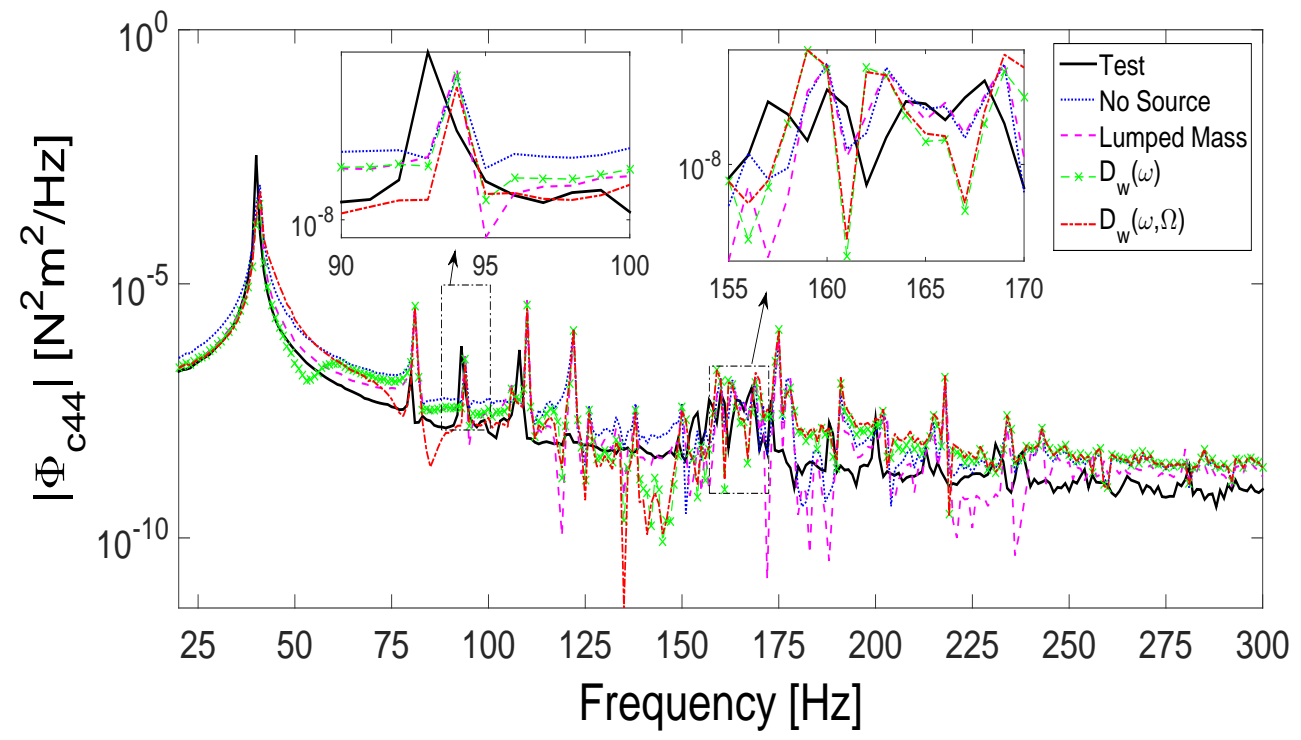

Fig. 16.

Comparison of the interface RWA-panel loads between test results and the four analytical approaches $(\Omega=2400 \mathrm{rpm})$ : (a) $\left|\Phi_{C 33}\right|$ and (b) $\left|\Phi_{C 44}\right|$. 


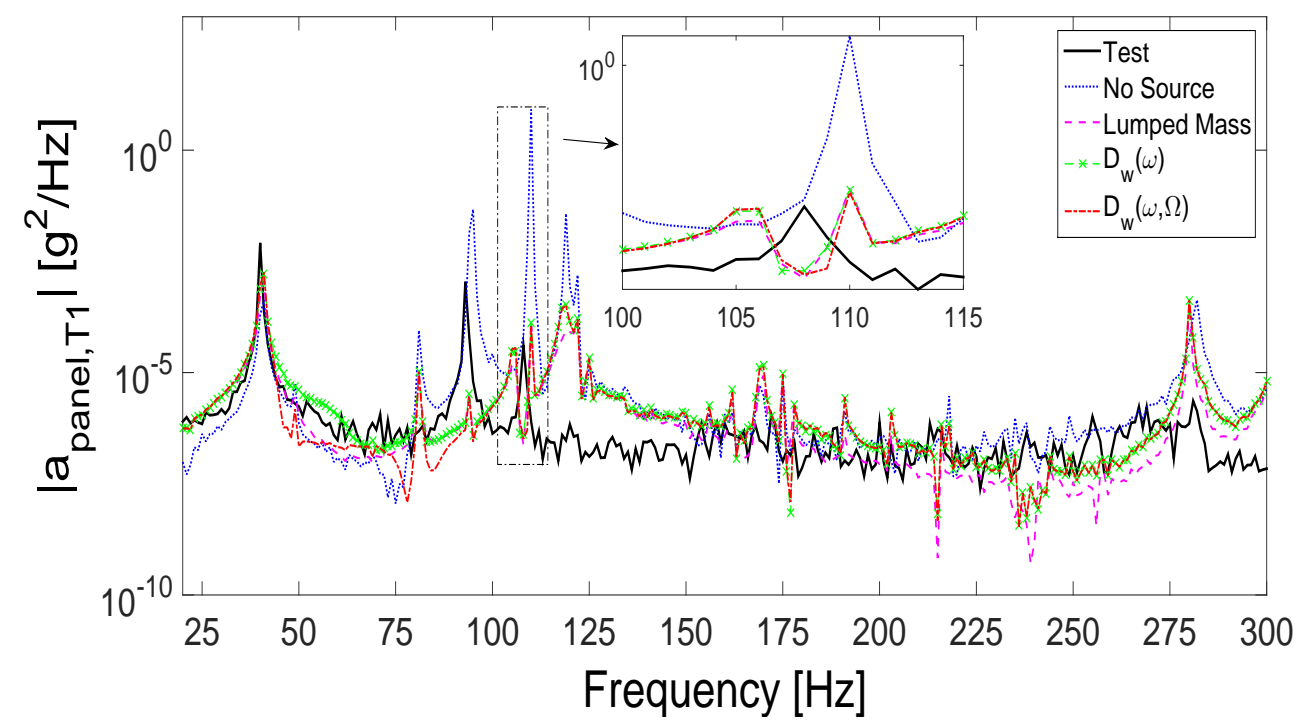

Fig. 17.

Comparison of the acceleration response of the panel between test results and the four analytical approaches $(\Omega=2400 \mathrm{rpm})$.

to lack of RWA dynamics implemented in the model. In contrast, models (c) and (d) presented an increased agreement throughout the frequency band of interest. The latter, in particular, was not only able to reproduce the coupled dynamics between the RWA and the panel (as model (c) did too) but was also able to accurately simulate the RWA structural dynamics. This is remarked in Table 2 where the analytical outcomes are expressed in terms of FRAC using Eq. (8) to show the beneficial effect of considering the RWA and the supporting structure dynamic mass matrices when dealing with coupled analysis. At frequencies beyond $200 \mathrm{~Hz}$ however, the coupled microvibrations predicted when implementing the RWA dynamic mass and that obtained by representing the RWA as a lumped mass are similar. This is explained due to absence of RWA modes in that region. The outcomes presented in this 
Table 2.

FRAC calculated for the RWA-panel coupled system responses

\begin{tabular}{lccc}
\hline & Model (a) & Model (c) & Model (d) \\
\hline \hline$\Phi_{C 33}$ & 0.2655 & 0.4304 & 0.4502 \\
$\Phi_{C 44}$ & 0.4389 & 0.4391 & 0.4618 \\
$a_{\text {panel }, T 1}$ & 0.2984 & 0.4282 & 0.4424 \\
\hline
\end{tabular}

section asserts that the method of implementing the RWA dynamic mass including the gyroscopic effect, is a valid and more favorable methodology for coupled microvibration analysis w.r.t. the traditional method where the hard-mounted forces and moments are directly applied to the supporting structure. Moreover, it offers an improved prediction over the traditional RWA static dynamic mass. In addition, it was observed that if the resonances of the supporting structure are well beyond the frequency band of interest, the advantage of using RWA dynamic mass is strictly limited to the region where the RWA modes are important.

\section{Conclusions}

The coupled microvibrations dynamics when a cantilever-configured RWA is mounted on a supporting structure were described in this article. Two coupled setups were developed to measure the reaction loads at the interface between the supporting structures and the RWA and in a coupled boundary condition with the bodies suspended free-free. Moreover, the response of the structures at multiple locations were also evaluated. The first test configuration implemented a rigid structure of which the modes were beyond the 
frequency band of interest whereas the second considered a flexible structure with modes occurring between $20 \mathrm{~Hz}$ and $300 \mathrm{~Hz}$. The former was primarily used as a benchmark example to validate the RWA microvibration model. The predictions derived from the traditional approach that simply uses the forces and moments from the hard-mounted boundary condition as direct input to the supporting structures has been demonstrated to ignore significant characteristics of the system response. It was also observed that the application of correcting factors such as the RWA and supporting structure dynamic masses, considerably improved the agreement between analytical estimates and test results. Finally, the inclusion of the gyroscopic effect in the RWA internal dynamics characterisation, proved to offer superior predictions over the aforementioned approach. Practically, the dynamic mass of a RWA including the gyroscopic effect can be derived from its analytical model, provided that this is available and validated. Thereby, the analysis process can be conducted following a systematic approach for any type of RWA. 


\section{Appendix A. RWA Imbalance Flywheel System Matrices}

With reference to Eq.(1), all matrices are as follows:

$$
\begin{gathered}
\mathbf{M}_{w a}=\operatorname{diag}\left\{M_{w}, M_{w}, M_{w}, I_{R_{w}}, I_{R_{w}}, M_{b}, M_{b}, M_{b}, I_{R_{b}}, I_{R_{b}}\right\} \\
\mathbf{G}_{w a}=\left\{\begin{array}{l}
G_{w a 45}=\Omega I_{Z_{w}} \\
G_{w a 54}=-\Omega I_{Z_{w}} \\
0, \quad \text { elsewhere }
\end{array}\right.
\end{gathered}
$$




$$
\begin{aligned}
& \mathbf{C}_{w a}=\left[\begin{array}{cccccc}
c_{T_{w}} & 0 & 0 & 0 & -c_{T_{w}} d & -c_{T_{w}} \\
0 & c_{T_{w}} & 0 & c_{T_{w}} d & 0 & 0 \\
0 & 0 & c_{z_{w}} & 0 & 0 & 0 \\
0 & c_{T_{w}} d & 0 & c_{T_{w}} d^{2}+c_{r_{w}} & 0 & \\
-c_{T_{w}} d & 0 & 0 & 0 & c_{T_{w}} d^{2}+c_{r_{w}} & c_{T_{w}} d \\
-c_{T_{w}} & 0 & 0 & 0 & c_{T_{w}} d & c_{T_{w}}+c_{T_{b}} \\
0 & -c_{T_{w}} & 0 & -c_{T_{w}} d & 0 & 0 \\
0 & 0 & -c_{z_{w}} & 0 & 0 & 0 \\
0 & c_{T_{w}} e & 0 & c_{T_{w}} d e-c_{r_{w}} & 0 & 0 \\
-c_{T_{w}} e & 0 & 0 & 0 & c_{T_{w}} d e-c_{r_{w}} & \left(c_{T_{w}}-c_{T_{b}}\right) e
\end{array}\right. \\
& 0 \quad 0 \quad-\quad-c_{T_{w}} e
\end{aligned}
$$

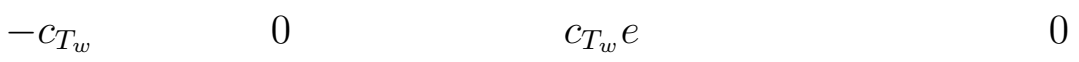

$$
\begin{aligned}
& \begin{array}{lll}
0-c_{z_{w}} & 0 & 0
\end{array}
\end{aligned}
$$

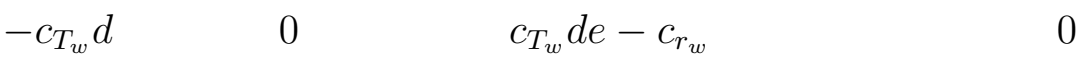

$$
\begin{aligned}
& 0 \quad 0 \quad c_{T_{w}} d e-c_{r_{w}} \\
& \begin{array}{lllll}
\cdots & 0 & 0 & 0 & \left(c_{T_{w}}-c_{T_{b}}\right) e
\end{array} \\
& \begin{array}{cclc}
c_{T_{w}}+c_{T_{b}} & 0 & -\left(c_{T_{w}}-c_{T_{b}}\right) e & 0
\end{array} \\
& \begin{array}{lll}
0 & c_{z_{w}}+c_{z_{b}} & 0
\end{array} \\
& -\left(c_{T_{w}}-c_{b_{w}}\right) e \quad 0 \quad\left(c_{T_{w}}+c_{T_{b}}\right) e^{2}+c_{r_{w}}+c_{r_{b}} \quad 0 \\
& 0 \\
& 0 \\
& 0 \\
& \left(c_{T_{w}}+c_{T_{b}}\right) e^{2}+c_{r_{w}}+c_{r_{b}}
\end{aligned}
$$




$$
\begin{aligned}
& \mathbf{K}_{w a}=\left[\begin{array}{cccccc}
k_{T_{w}} & 0 & 0 & 0 & -k_{T_{w}} d & -k_{T_{w}} \\
0 & k_{T_{w}} & 0 & k_{T_{w}} d & 0 & 0 \\
0 & 0 & k_{z_{w}} & 0 & 0 & 0 \\
0 & k_{T_{w}} d & 0 & k_{T_{w}} d^{2}+k_{r_{w}} & 0 & \\
-k_{T_{w}} d & 0 & 0 & 0 & k_{T_{w}} d^{2}+k_{r_{w}} & k_{T_{w}} d \\
-k_{T_{w}} & 0 & 0 & 0 & k_{T_{w}} d & k_{T_{w}}+k_{T_{b}} \\
0 & -k_{T_{w}} & 0 & -k_{T_{w}} d & 0 & 0 \\
0 & 0 & -k_{z_{w}} & 0 & 0 & 0 \\
0 & k_{T_{w}} e & 0 & k_{T_{w}} d e-k_{r_{w}} & 0 & 0 \\
-k_{T_{w}} e & 0 & 0 & 0 & k_{T_{w}} d e-k_{r_{w}} & \left(k_{T_{w}}-k_{T_{b}}\right) e
\end{array}\right. \\
& 0 \quad 0 \quad 0 \quad-k_{T_{w}} e \\
& \begin{array}{llll}
-k_{T_{w}} & 0 & k_{T_{w}} e & 0
\end{array} \\
& \begin{array}{lll}
0 & -k_{z_{w}} & 0
\end{array}
\end{aligned}
$$

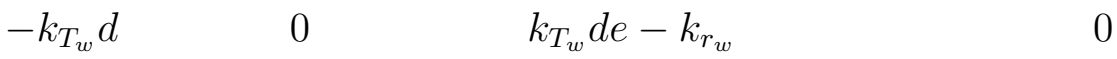

$$
\begin{aligned}
& 0 \quad 0 \quad k_{T_{w}} d e-k_{r_{w}} \\
& \begin{array}{lllll}
\cdots & 0 & 0 & 0 & \left(k_{T_{w}}-k_{T_{b}}\right) e
\end{array}
\end{aligned}
$$

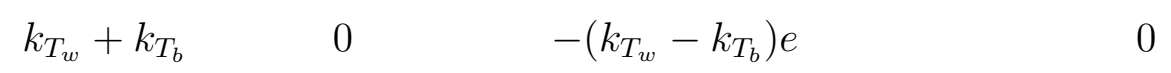

$$
\begin{aligned}
& 0 \quad k_{z_{w}}+k_{z_{b}} \quad 0 \quad 0 \\
& -\left(k_{T_{w}}-k_{b_{w}}\right) e \quad 0 \quad\left(k_{T_{w}}+k_{T_{b}}\right) e^{2}+k_{r_{w}}+k_{r_{b}} \quad 0 \\
& 0 \quad 0 \quad \begin{array}{lll}
0 & \left(k_{T_{w}}+k_{T_{b}}\right) e^{2}+k_{r_{w}}+k_{r_{b}}
\end{array}
\end{aligned}
$$




$$
\mathbf{q}_{w a}=\left[\begin{array}{c}
x_{w} \\
y_{w} \\
z_{w} \\
\theta_{w} \\
\varphi_{w} \\
x_{b} \\
y_{b} \\
z_{b} \\
\theta_{b} \\
\varphi_{b}
\end{array}\right] \quad \mathbf{f}_{w a}=\left[\begin{array}{l}
\sum_{j=1}^{n_{T}} A_{j}^{T} \Omega^{2} \sin \left(H_{j}^{T} t\right)+U_{T}(\Omega) \\
\sum_{j=1}^{n_{T}} A_{j}^{T} \Omega^{2} \cos \left(H_{j}^{T} t\right)+U_{T}(\Omega) \\
\sum_{j=1}^{n_{Z}} A_{j}^{Z} \Omega^{2} \sin \left(H_{j}^{Z} t\right)+U_{Z}(\Omega) \\
\sum_{j=1}^{n_{R}} A_{j}^{R} \Omega^{2} \cos \left(H_{j}^{R} t\right)+U_{R}(\Omega) \\
0 \\
0 \\
0 \\
0 \\
0
\end{array}\right]
$$

The superscripts $T, R$ and $Z$ in the excitation vector indicate translational, rotational and axial DoFs respectively. The harmonic number is represented by $H$, which is a fraction of the excitation speed $\Omega$, whereas $A$ is the amplitude of $j$-th harmonic considered in the model. The total number of harmonics in the model is assumed equal to $n$. Finally, $U(\Omega)$ represents the broadband noise in function of the excitation speed.

\section{References}

[1] O. L. de Weck, D. W. Miller, H. Guiterrez, Structural dynamics and control for ngst - a preliminary study, in: The 34th Lige International Astrophysics Colloquium, Lige, Belgium, 1998.

[2] T. T. Hyde, K. Q. Ha, J. D. Johnston, J. M. Howard, G. E. Mosier, Integrated modeling activities for the james webb space telescope: Op- 
tical jitter analysis, in: SPIE Conference on Astronomical Telescopes and Instrumentation, Glasgow, Scotland, 2004.

[3] Y. Katsukawa, Y. Masada, T. Shimizu, S. Sakai, K. Ichimoto, Pointing stability of hinode and requirements for the next solar mission solar-c, in: International Conference on Space Optics, Rhodes, Greece, 2010.

[4] M. Toyoshima, Y. Takayama, H. Kunimori, T. Jono, S. Yamakawa, Inorbit measurements of spacecraft microvibrations for satellite laser communication links, Optical Engineering 49 (8) (2010) 083604-10.

[5] T. Wacker, L. Weimer, K. Eckert, Goce platform micro-vibration verification by test and analysis, in: European Conference on Spacecraft Structures, Materials \& Mechanical Testing, Noordwijk, The Netherlands, 2005.

[6] E. Koekkoek, P. Gungo, R. Bureo Dacal, A realistic approach in the prediction of the aces micro-gravity environment, in: European Conference on Spacecraft Structures, Materials \& Mechanical Testing, Noordwijk, The Netherlands, 2005.

[7] F. Deng, X. He, J. Zhang, L. Li, X. Dong, Research on the effects of microgravity vibration enviroment in the spacelab, Journal of Vibration and Shock 24 (3) (2005) 103-107.

[8] M. Remedia, G. Aglietti, G. Richardson, A stochastic methodology for predictions of the environment created by multiple microvibration sources, Journal of Sound and Vibration 344 (2015) 138-157. doi:10.1016/j.jsv.2015.01.035. 
[9] M. Remedia, G. Aglietti, G. Richardson, M. Sweeting, Integrated semiempirical methodology for microvibration prediction, AIAA Journal 53 (5) (2015) 1236-1250. doi:10.2514/1.J053339.

[10] W. Desmet, B. Pluymers, O. Atak, MID-FREQUENCY - CAE Methodologies for mid-frequency analysis in vibrations and acoustics, 2012.

[11] ECSS-E-HB-32-26A Spacecraft Mechanical Loads Analysis Handbook, ESA Requirements and Standards Division, 2013.

[12] B. Bialke, Microvibration disturbance fundamentals for rotating mechanisms, in: 34th Annual Guidance and Control Conference, Breckenridge, CO, 2011.

[13] S. E. Miller, P. Kirchman, J. Sudey, Reaction wheel operational impacts on the goes-n jitter environment, in: AIAA Guidance, Navigation and Control Conference and Exhibit, South Carolina, USA, 2007.

[14] O. Takahara, N. Yoshida, K. Minesugi, Microvibration transmissibility test of solar-b, in: The 24th International Symposium on Space Technology and Science, Miyazaki, Japan, 2004.

[15] O. Takahara, K. Ichimoto, T. Kosugi, S. Shimada, N. Yoshida, Evaluation of pointing error for solar-b using optical measurement, Uchu Kagaku Gijutsu Rengo Koenkai Koenshu 50 (2006) 271-275.

[16] L. Vaillon, B. Sanctorum, J. Sperandei, A. Defendini, G. Griseri, P. Spanoudakis, M. von Alberti, Flight prototyping of active control 
of vibration \& very high accuracy pointing systems, in: 5th International ESA Conference on Guidance Navigation and Control Systems, Rome, Italy, 2002.

[17] J.-S. Bae, J.-H. Hwang, J.-H. Roh, J.-H. Kim, M.-S. Yi, J.-H. Lim, Vibration suppression of a cantilever beam using magnetically tunedmass-damper, Journal of Sound and Vibration 331 (26) (2012) 5669 5684. doi:10.1016/j.jsv.2012.07.020.

[18] B. Bialke, Microvibration disturbance sources in reaction wheels and momentum wheels, in: European Conference on Spacecraft Structures, Materials \& Mechanical Testing, Noordwijk, The Netherlands, 1996.

[19] R. A. Masterson, D. W. Miller, R. L. Grogan, Development and validation of reaction wheel disturbance models: Empirical model, Journal of Sound and Vibration 249 (3) (2002) 575-598.

[20] L. M. Elias, F. G. Dekens, I. Basdogan, L. A. Sievers, T. Neville, A methodology for modeling the mechanical interaction between a reaction wheel and a flexible structure, in: Interferometry in Space, Waikoloa, HI, USA, 2003.

[21] Z. Zhang, G. Aglietti, Microvibration modelling and testing of a satellite reaction wheel, in: European Conference of Mechanical Engineering (ECME'11), Puerto De La Cruz, Tenerife, 2011.

[22] P. Laurens, E. Decoux, M. Janvier, Soho microvibrations: Analyses, tests and flight results, in: Spacecraft Guidance, Navigation and Control 
Systems, Proceedings of the 3rd ESA International Conference, Noordwijk, the Netherlands, 1997.

[23] L. M. Elias, D. W. Miller, A coupled disturbance analysis method using dynamic mass measurement techniques, in: 43rd AIAA/ASME/ASCE/AHS/ASC Structures, Structural Dynamics, and Materials Conference, Denver, Colorado, 2002.

[24] S. Taniwaki, Y. Ohkami, Experimental and numerical analysis of reaction wheel disturbances, JSME International Journal Series C Mechanical Systems, Machine Elements and Manufacturing 46 (2) (2003) $519-526$.

[25] S. Taniwaki, M. Kudo, M. Sato, Y. Ohkami, Analysis of retainer induced disturbances of reaction wheel, Journal of System Design and Dynamics 1 (2) (2007) 307-317.

[26] K.-C. Liu, P. Maghami, C. Blaurock, Reaction wheel disturbance modeling, jitter analysis, and validation tests for solar dynamics observatory, in: AIAA Guidance, Navigation and Control Conference and Exhibit, Honolulu, Hawaii, 2008.

[27] P. Marucchi-Chierro, C. Galeazzi, The microvibration environment on artemis : A strategy for its characterization and control, in: 46th International Astronautical Congress, Oslo, Norway, 1995.

[28] O. L. de Weck, D. W. Miller, Integrated modeling and dynamics simulation for the next generation space telescope, Ph.D. thesis (1999). 
[29] Z. Zhang, W. Ren, G. Aglietti, Coupled disturbance modelling and validation of a reaction wheel model, in: European Conference on Spacecraft Structures, Materials \& Environmental Testing, The Netherlands, Noordwijk, 2012.

[30] Y. Zhao, P. Zhang, W. Cheng, Measurement and study of disturbance characteristics of reaction wheel assembly, Journal of Experimental Mechanics 24 (6) (2009) 532-538.

[31] Z. Zhang, G. S. Aglietti, W. Ren, Coupled microvibration analysis of a reaction wheel assembly including gyroscopic effects in its accelerance, Journal of Sound and Vibration 332 (22) (2013) 5748-5765.

[32] C. Zang, H. Grafe, M. Imregun, Frequency-domain criteria for correlation and updating finite element models, Mechanical Systems and Signal Processing 15 (2001) 139-155. doi:10.1006/mssp.2000.1357.

[33] Z. Zhang, G. Aglietti, W. Zhou, Microvibrations induced by a cantilevered wheel assembly with a soft-suspension system, AIAA Journal 49 (5) (2011) 1067-1079.

[34] D. Addari, G. Aglietti, M. Remedia, Investigating microvibration sources modelling, in: 13th European Conference on Spacecraft Structures, Materials and Environmental Testing, Braunschweig, Germany, 2014 .

[35] M. Hazewinkel, Encyclopedia of Mathematics: an updated and annotated translation of the Soviet Mathematical Encyclopedia, Springer Netherlands, 1997. 
[36] P. Laurens, E. Decoux, Understanding and monitoring space mechanisms through their microdynamic signature, in: The 7th European Space Mechanisms and Tribology Symposium, Noordwijk, Netherlands, 1997.

[37] L. M. Elias, D. W. Miller, Dynamics of multi-body space interferometers including reaction wheel gyroscopic stiffening effects: Structurally connected and electromagnetic formation flying architectures, Ph.D. thesis (2004).

[38] I. Basdogan, L. M. Elias, F. Dekens, L. Sievers, Predicting the optical performance of the space interferometry mission using a modeling, testing, and validation, Journal of Vibration and Acoustics 129 (2) (2007) 148-157.

[39] D. Addari, G. Aglietti, M. Remedia, G. Richardson, B. LePage, Inspecting the characterisation of microvibration sources, in: 65th International Astronautical Congress, Toronto, Canada, 2014.

[40] Z. Zhang, G. Aglietti, W. Ren, Microvibration model development and validation of a cantilevered reaction wheel assembly, Applied Mechanics and Materials 226-228 (2012) 133-137.

[41] M. Remedia, G. Aglietti, Modelling the effect of electrical harness on microvibration response of structures, Acta Astronautica 109 (2015) 88102. doi:10.1016/j.actaastro.2014.12.017.

[42] E. Swanson, C. D. Powell, S. Weissman, A practical review of rotating 
machinery critical speeds and modes, Sound and vibration 39 (5) (2005) 16-17. 